TRANSACTIONS OF THE

AMERICAN MATHEMATICAL SOCIETY

Volume 359, Number 11, November 2007, Pages 5141-5170

S 0002-9947(07)04460-1

Article electronically published on June 4, 2007

\title{
THE HAUSDORFF DIMENSION OF VISIBLE SETS OF PLANAR CONTINUA
}

\author{
TOBY C. O'NEIL
}

\begin{abstract}
For a compact set $\Gamma \subset \mathbb{R}^{2}$ and a point $x$, we define the visible part of $\Gamma$ from $x$ to be the set

$$
\Gamma_{x}=\{u \in \Gamma:[x, u] \cap \Gamma=\{u\}\} .
$$

(Here $[x, u]$ denotes the closed line segment joining $x$ to $u$.)

In this paper, we use energies to show that if $\Gamma$ is a compact connected set of Hausdorff dimension greater than one, then for (Lebesgue) almost every point $x \in \mathbb{R}^{2}$, the Hausdorff dimension of $\Gamma_{x}$ is strictly less than the Hausdorff dimension of $\Gamma$. In fact, for almost every $x$,
\end{abstract}

$$
\operatorname{dim}_{H}\left(\Gamma_{x}\right) \leq \frac{1}{2}+\sqrt{\operatorname{dim}_{H}(\Gamma)-\frac{3}{4}} .
$$

We also give an estimate of the Hausdorff dimension of those points where the visible set has dimension greater than $\sigma+\frac{1}{2}+\sqrt{\operatorname{dim}_{H}(\Gamma)-\frac{3}{4}}$ for some $\sigma>0$.

\section{INTRODUCTION}

Given a subset $E$ of the plane, Urysohn [11, 12] defined the notion of linear accessibility for a point $p \in E: p$ is linearly accessible if there is a non-degenerate line segment $L$ that only meets $E$ at the point $p$. In a sequence of papers, Nikodym 7 , [8, 9] investigated the relationship between the set theoretic complexity of $E$ and the set of linearly accessible points.

In this paper, we consider those points of a planar continuum (a compact connected subset of the plane) $\Gamma$ that are linearly accessible from a given fixed point $x$ and investigate the relationship between the (Hausdorff) dimensions of the compact set and its linearly accessible part from $x$ for Lebesgue almost all $x \in \mathbb{R}^{2} \backslash \Gamma$. Denoting $\Gamma_{x}$ to be the points of $\Gamma$ that are linearly accessible from $x$, it is clear that $\operatorname{dim}_{H}\left(\Gamma_{x}\right) \leq \operatorname{dim}_{H}(\Gamma)$ for all $x \in \mathbb{R}^{2} \backslash \Gamma$. However, more surprisingly, for most points there is a drop in dimension.

Proceeding more formally, if for a compact set in the plane, $K$, and $x \in \mathbb{R}^{2}$ we define the visible part of $K$ from $x$ by

$$
K_{x}=\{u \in K:[x, u] \cap K=\{u\}\},
$$

where $[x, u]$ denotes the closed line segment joining $x$ to $u$, then our results may be summarised as follows.

Received by the editors November 6, 2003.

2000 Mathematics Subject Classification. Primary 28A80; Secondary 28A78, 31A15.

Key words and phrases. Visible sets, Hausdorff dimension.

(C)2007 American Mathematical Society 
Theorem 1.1. If $\Gamma$ is a planar continuum with $\operatorname{dim}_{H}(\Gamma) \geq 1$, then for (Lebesgue) almost all $x \in \mathbb{R}^{2}$,

$$
\operatorname{dim}_{H}\left(\Gamma_{x}\right) \leq \frac{1}{2}+\sqrt{\operatorname{dim}_{H}(\Gamma)-\frac{3}{4}}
$$

This follows directly from the stronger theorem that we prove in this paper.

Theorem 1.2. Let $\Gamma$ be a planar continuum with $\operatorname{dim}_{H}(\Gamma)>1$. Then for $\frac{1}{2}+$ $\sqrt{\operatorname{dim}_{H}(\Gamma)-\frac{3}{4}}<s \leq \operatorname{dim}_{H}(\Gamma)$,

$$
\operatorname{dim}_{H}\left\{x \in \mathbb{R}^{2}: \operatorname{dim}_{H}\left(\Gamma_{x}\right)>s\right\} \leq \frac{\operatorname{dim}_{H}(\Gamma)-s}{s-1} .
$$

(To see how Theorem 1.1 follows from Theorem 1.2, first observe that if $\operatorname{dim}_{H}(\Gamma)$ $=1$, then Theorem 1.1 is obviously true. If $d=\operatorname{dim}_{H}(\Gamma)>1$, then Theorem 1.1 follows provided we show that for $\sigma>0$,

$$
\operatorname{dim}_{H}\left\{x \in \mathbb{R}^{2}: \operatorname{dim}_{H}\left(\Gamma_{x}\right)>\sigma+\frac{1}{2}+\sqrt{d-\frac{3}{4}}\right\}<2 .
$$

However, $1<\frac{1}{2}+\sqrt{d-\frac{3}{4}}<d$, and Theorem[1.2 implies that for $s \in\left(\frac{1}{2}+\sqrt{d-\frac{3}{4}}, d\right]$, $\operatorname{dim}_{H}\left\{x \in \mathbb{R}^{2}: \operatorname{dim}_{H}\left(\Gamma_{x}\right)>s\right\} \leq \frac{d-s}{s-1}$. An easy algebraic manipulation then shows that

$$
\frac{d-s}{s-1}<\left(d-\frac{1}{2}-\sqrt{d-\frac{3}{4}}\right) /\left(-\frac{1}{2}+\sqrt{d-\frac{3}{4}}\right)<2,
$$

for $d \in(1,7)$.)

In an earlier paper [3, it is shown that for a particular class of continua (namely quasicircles), whenever $x$ lies outside the set, $\operatorname{dim}_{H}\left(\Gamma_{x}\right)=1$. Since quasicircles can have dimension arbitrarily close to 2 , and for connected sets of positive dimension, $\operatorname{dim}_{H}\left(\Gamma_{x}\right) \geq 1$ whenever $x \notin \Gamma$, it follows that, unless the optimal upper bound for $\operatorname{dim}_{H}\left(\Gamma_{x}\right)$ is one, there is no general result concerning the lower bound of $\operatorname{dim}_{H}\left(\Gamma_{x}\right)$ beyond the trivial estimate of one.

There are many possible directions for future work. Despite the fact that the upper bound given in Theorem 1.1 is the golden-ratio for $\operatorname{dim}_{H}(\Gamma)=2$, there is no good reason to believe that this bound is optimal, since the proof we give in this paper uses at least one sub-optimal estimate. Indeed, Theorem 1.2 gives no information on the size of the set of points where the visible sets have Hausdorff dimension between 1 and $\frac{1}{2}+\sqrt{\operatorname{dim}_{H}(\Gamma)-\frac{3}{4}}$ - it may be that there can be many points where the visible set has dimension between these bounds.

Our method of proving Theorem 1.2 relies in an essential way on the properties of connected sets in the plane, in particular, that the points visible from one location together with the connectedness of the original set result in certain regions of the plane not being visible from a different location. It is unclear whether a similar result could possibly hold in higher dimensions. Whether a dimension drop must occur for totally disconnected subsets of the plane is also unclear: in [3], it is shown that for the cross-product of a Cantor set with itself in the plane, there is a dimension drop (to 1), provided that the original Cantor set has Hausdorff dimension sufficiently close to 1 . 
I would like to thank Paul MacManus, Pertti Mattila and David Preiss for useful discussions during the writing of this paper, and Marianna Csörnyei for her comments on a preliminary draft of the paper. My thanks also go to the referee for helpful suggestions.

\section{BACKGROUND RESULTS AND PRELIMINARY ESTIMATES}

In this section we summarise the main definitions and results that we use.

Most of the time we shall be working in the plane, $\mathbb{R}^{2}$, endowed with the usual norm $|\cdot|$ and inner product $\langle\cdot, \cdot\rangle$. We let $e_{1}$ and $e_{2}$ denote the usual basis vectors in $\mathbb{R}^{2}$ and set $x^{\wedge}=x /|x|$ for $x \neq 0$, and $x^{\perp}=\left\langle x, e_{1}\right\rangle e_{2}-\left\langle x, e_{2}\right\rangle e_{1}$ for $x \in \mathbb{R}^{2}$. For $x \in \mathbb{R}^{2}$ and $A \subseteq \mathbb{R}^{2}$, define $\operatorname{arc}_{-\operatorname{diam}_{x}}(A)$ to be the angle (in radians) subtended by the smallest arc in the circle $\{u:|x-u|=1\}$ that contains the radial projection of $A$ onto this circle. (If $x \in A$, then $\operatorname{arc}_{-\operatorname{diam}_{x}}(A)=2 \pi$.) We let $\operatorname{diam}(A) \operatorname{denote}$ the usual diameter of a set $A$.

For a subset $A$ of the plane and $r>0$, let

$$
B(A, r)=\left\{y \in \mathbb{R}^{2}: \text { there is } x \in A \text { with }|y-x| \leq r\right\}
$$

and, in a slight abuse of notation, let $B(x, r)=B(\{x\}, r)$, the usual closed ball of centre $x$ and radius $r$. For a point $x$ and a set $A$, we define $d(x, A)=\inf \{|x-y|$ : $y \in A\}$.

Let $X$ be a Polish space. (That is, $X$ is a complete, separable, metrisable topological space.) A sub-additive, non-negative set function $\mu$ on $X$ is a Radon measure if it is a Borel measure (all Borel sets are $\mu$-measurable) for which all compact sets have finite measure and both

$$
\mu(U)=\sup \{\mu(K): K \subset U, K \text { is compact }\}, \text { for open sets } U
$$

and

$$
\mu(A)=\inf \{\mu(U): A \subset U, U \text { is open }\}, \text { for } A \subseteq X .
$$

We denote the set of Radon measures on $X$ by $\mathcal{M}(X)$.

We let $\sigma(\mathbf{A}(X))$ denote the $\sigma$-algebra generated by the analytic subsets of $X$; we suppress mention of $X$ when this is clear from the context. If $\mu$ is a Radon measure on this space, then all sets in $\sigma(\mathbf{A})$ are $\mu$-measurable; see [4, (21.10)].

For $s \in \mathbb{R}$ and $A \subseteq X$, we define

$$
\mathcal{M}^{s}(A)=\left\{\nu \in \mathcal{M}(X): \nu(A)>0 \text { and } \nu(B(x, r)) \leq r^{s} \text { for } x \in X \text { and } r>0\right\} .
$$

If $\mu$ is a Radon measure on the plane and $s \in \mathbb{R}$, then $I_{s}(\mu)$ denotes the $s$-energy of $\mu$ given by

$$
I_{s}(\mu)=\iint|x-y|^{-s} d \mu(x) d \mu(y) .
$$

The Hausdorff dimension of a set is defined in the usual way via Hausdorff measures; see 1, 2, 6, 10]. The following theorem summarises some useful equivalent ways of finding the Hausdorff dimension of a set.

Theorem 2.1. Let $A$ be an analytic subset of a Euclidean space $\mathbb{R}^{n}$. Then

$$
\begin{aligned}
\operatorname{dim}_{H}(A) & =\sup \left\{s \in \mathbb{R}: \mathcal{M}^{s}(A) \neq \emptyset\right\} \\
& =\sup \left\{s \in \mathbb{R}: \text { there is } \mu \in \mathcal{M}\left(\mathbb{R}^{n}\right) \text { with } \mu(A)>0 \text { and } I_{s}(\mu)<\infty\right\} \\
& =\sup \left\{\operatorname{dim}_{H}(K): K \subseteq A \text { and } K \text { is compact }\right\} .
\end{aligned}
$$


Proof. See [6, Theorem 8.8] and [1, Theorem 6.4] together with [2, 2.10.48] or [10, Theorem 57].

2.1. Elementary geometric estimates. We end this subsection by recording some simple geometric estimates that we use later.

For $x \in \mathbb{R}^{2}, u \in \mathbb{R}^{2} \backslash\{0\}$ and $\sigma>0$, let

$$
V(x, u, \sigma)=\left\{y \in \mathbb{R}^{2}:\left|\left\langle y-x, u^{\perp}\right\rangle\right|<\sigma\langle y-x, u\rangle\right\},
$$

the open cone with vertex $x$, direction $u$ and opening $\sigma$. The following lemma gives a lower bound on the distance of a point in a particular subregion of a cone from the vertex.

Lemma 2.2. Let $p \in \mathbb{R}^{2} \backslash\{0\}$ and $\sigma, \tau>0$. If

$$
u \in V(0, p, \sigma) \backslash V(p,-p, \tau),
$$

then

$$
\left\langle u-p, p^{\wedge}\right\rangle \geq-\frac{\sigma}{\sigma+\tau}|p| .
$$

Proof. Suppose that $u \in V(0, p, \sigma) \backslash V(p,-p, \tau)$. Then

$$
\left\langle u-p, p^{\wedge}\right\rangle \geq\left\langle q-p, p^{\wedge}\right\rangle
$$

where

$$
q=\mu\left(p+\sigma p^{\perp}\right)=p+\lambda\left(-p+\tau p^{\perp}\right),
$$

for some $\mu, \lambda>0$. Calculating $\left\langle q, p^{\perp}\right\rangle$ gives

$$
\mu=\lambda \frac{\tau}{\sigma}
$$

and substituting for $\mu$ in $\langle q, p\rangle$ gives

$$
\lambda=\frac{\sigma}{\sigma+\tau} .
$$

Hence

$$
\left\langle q-p, p^{\wedge}\right\rangle \geq-\frac{\sigma}{\sigma+\tau}|p|
$$

as required.

The second lemma is slightly trickier to prove and is an estimate of the angle subtended by the points $p-a$ and $p+a$ with the origin when $a$ and $p$ are sufficiently separated. For $x \in \mathbb{R}^{2}, d_{-}, d_{+} \in \mathbb{R}^{+}$, let $A\left(x, d_{-}, d_{+}\right)=B\left(x, d_{+}\right) \backslash B\left(x, d_{-}\right)$, the half-open annulus centred on $x$ with inner radius $d_{-}$and outer radius $d_{+}$.

Lemma 2.3. Suppose that $0<d_{-} \leq d_{+}, a \in B\left(0, \frac{1}{2} d_{-}\right) \backslash\{0\}$ and $E \subseteq A\left(0, d_{-}, d_{+}\right)$ is a compact set. Let $\alpha=\min \left\{\left|\left\langle p, a^{\perp}\right\rangle /\langle p, a\rangle\right|: p \in E\right\}$. If $\alpha \leq 1$, then for all $p \in E$,

$$
\frac{3}{5} \leq \frac{\langle p-a, p+a\rangle}{|p-a||p+a|} \leq 1-\frac{9}{17 d_{+}^{2}}(|a| \alpha)^{2} .
$$

Proof. For any $p \in \mathbb{R}^{2}$,

$$
\langle p-a, p+a\rangle=|p|^{2}-|a|^{2}
$$

and

$$
|p-a|^{2}|p+a|^{2}=\left(|p|^{2}+|a|^{2}\right)^{2}-4\langle p, a\rangle^{2} .
$$


On defining $A=\left\langle p, a^{\perp}\right\rangle /\langle p, a\rangle$ (possibly infinite), then $1+A^{2}=\frac{|p|^{2}|a|^{2}}{\langle p, a\rangle^{2}}$, and so

$$
|p-a|^{2}|p+a|^{2}=\frac{\left(|p|^{2}-|a|^{2}\right)^{2}+\left(|p|^{2}+|a|^{2}\right)^{2} A^{2}}{1+A^{2}} .
$$

(We take $|p-a|^{2}|p+a|^{2}=\left(|p|^{2}+|a|^{2}\right)^{2}$ for $|A|=+\infty$.) Hence

$$
\left(\frac{\langle p-a, p+a\rangle}{|p-a||p+a|}\right)^{2}=\frac{1+A^{2}}{1+(1+\mu)^{2} A^{2}}=1-\frac{\mu(2+\mu) A^{2}}{1+(1+\mu)^{2} A^{2}},
$$

where

$$
\mu=\frac{2(|a| /|p|)^{2}}{1-(|a| /|p|)^{2}}
$$

Expression (*) is a monotonic decreasing function of $A$. Hence for $p \in E$, $(*)$ is maximised when $A=\alpha$, and so, since $\frac{\mu(2+\mu) \alpha^{2}}{1+(1+\mu)^{2} \alpha^{2}}=1-\frac{1+\alpha^{2}}{1+(1+\mu)^{2} \alpha^{2}} \in[0,1]$, we use that for $0 \leq x \leq 1,(1-x)^{\frac{1}{2}} \leq 1-\frac{1}{2} x$ to deduce

$$
\frac{\langle p-a, p+a\rangle}{|p-a||p+a|} \leq 1-\frac{1}{2}\left(\frac{\mu(2+\mu) \alpha^{2}}{1+(1+\mu)^{2} \alpha^{2}}\right) \text {. }
$$

However

$$
0<2 \frac{|a|^{2}}{d_{+}^{2}} \leq 2\left(\frac{|a|}{|p|}\right)^{2} \leq \mu=\frac{2(|a| /|p|)^{2}}{1-(|a| /|p|)^{2}} \leq \frac{8}{3}\left(\frac{|a|}{|p|}\right)^{2} \leq \frac{2}{3},
$$

and so $(2+\mu) /\left(1+(1+\mu)^{2} \alpha^{2}\right) \geq \frac{9}{17}$, giving

$$
\frac{\langle p-a, p+a\rangle}{|p-a||p+a|} \leq 1-\frac{9}{34} \mu \alpha^{2} \leq 1-\frac{9}{17 d_{+}^{2}}(|a| \alpha)^{2} .
$$

The lower bound follows from recognising that $\left(^{*}\right)$ is minimised when $p=$ $d_{-} a^{\perp} /|a|$, giving $A=+\infty, \mu=2|a|^{2} /\left(d_{-}^{2}-|a|^{2}\right)$ and

$$
\left(\frac{\langle p-a, p+a\rangle}{|p-a||p+a|}\right)^{2}=\frac{1}{(1+\mu)^{2}} \geq\left(\frac{3}{5}\right)^{2} .
$$

2.2. Elementary measure estimates. We now prove some estimates concerning the geometric distribution of mass for Radon measures in the plane.

We start by recording a simple mass estimate.

Lemma 2.4. Fix $s>0$ and $0<d_{+}$. Let $\nu$ be a Radon measure such that for all $u \in \mathbb{R}^{2}$ and $r>0, \nu(B(u, r)) \leq r^{s}$. Suppose that $x \in \mathbb{R}^{2}$ and $V \subseteq \mathbb{R}^{2}$. Then

$$
\nu\left(V \cap B\left(x, d_{+}\right)\right) \leq c \operatorname{arc-diam}_{x}\left(V \cap B\left(x, d_{+}\right)\right)^{s-1},
$$

for some fixed positive constant $c$ depending only on $d_{+}$and $s$.

Proof. We may suppose that $x=0$. Let $\theta=\operatorname{arc-diam}_{0}(V)$. If $\theta \leq 1 / 2$, then $V \cap A\left(0, d_{-}, d_{+}\right)$may be covered by $1+\frac{d_{+}}{d_{+} \theta}$ boxes of side $d_{+} \theta$. Hence a simple estimate of mass gives

$$
\nu\left(V \cap B\left(0, d_{+}\right)\right) \leq 2^{\frac{1}{2} s}\left(1+\theta^{-1}\right)\left(d_{+} \theta\right)^{s} \leq 3 d_{+}^{s} 2^{\frac{1}{2} s-1} \theta^{s-1},
$$

and the lemma follows for $\theta \leq 1 / 2$. If $\theta \geq 1 / 2$, then we use the estimate that $\nu\left(B\left(0, d_{+}\right)\right) \leq d_{+}^{s}$. 
We now prove a lemma on the distribution of mass for an arbitrary measure in semi-infinite tubes. To do this we define for $x \in \mathbb{R}^{2}$ and $r>0$,

$$
T^{+}(x, r)=\left\{z \in \mathbb{R}^{2}:\left|p_{1}(z)-p_{1}(x)\right|<r \text { and } p_{2}(z) \geq p_{2}(x)\right\}
$$

and

$$
T^{-}(x, r)=\left\{z \in \mathbb{R}^{2}:\left|p_{1}(z)-p_{1}(x)\right|<r \text { and } p_{2}(z) \leq p_{2}(x)\right\},
$$

where $p_{1}$ and $p_{2}$ denote orthogonal projections onto the $x$ - and $y$-axis, respectively. Thus $T^{+}(x, r)$ is a semi-open vertical tube of width $2 r$ extending upwards from $x$ and $T^{-}(x, r)$ is a semi-open vertical tube of width $2 r$ extending downwards from $x$.

Proposition 2.5. Suppose $\nu$ is a compactly supported Radon measure in the plane. Then for $\xi>0$ and $\nu$-a.e. $x$

$$
\liminf _{r \rightarrow 0} \frac{\nu\left(T^{+}(x, r)\right)}{r^{1+\xi}}=\liminf _{r \rightarrow 0} \frac{\nu\left(T^{-}(x, r)\right)}{r^{1+\xi}}=+\infty .
$$

Proof. We give the proof for $T^{+}$; the proof for $T^{-}$is similar. Without loss of generality we can assume that $\operatorname{spt} \nu$ lies in the unit square $[0,1] \times[0,1]$.

For $M \in \mathbb{N}$ and $i \in \mathbb{N}$, let

$$
A_{M, i}=\left\{x \in[0,1] \times[0,1]: \nu\left(T^{+}\left(x, 2^{-i}\right)\right) \leq M 2^{-i(1+\xi)}\right\},
$$

and define

$$
B_{M, i}=\bigcup_{x \in A_{M, i}}\left(\left[x-2^{-(i+1)}, x+2^{-(i+1)}\right] \times[-1,1]\right) .
$$

Notice that for each $z \in B_{M, i}, \nu\left(A_{M, i} \cap T^{+}\left(z, 2^{-(i+1)}\right)\right) \leq M 2^{-i(1+\xi)}$ : for if $A_{M, i} \cap$ $T^{+}\left(z, 2^{-(i+1)}\right)=\emptyset$, then there is nothing to prove; otherwise if we set

$$
H^{+}=T^{+}\left(z, 2^{-(i+1)}\right) \cap \bigcup\left\{T^{+}\left(x, 2^{-i}\right): x \in A_{M, i} \cap T^{+}\left(z, 2^{-(i+1)}\right)\right\},
$$

then $\nu\left(A_{M, i} \cap T^{+}\left(z, 2^{-(i+1)}\right)\right) \leq \nu\left(H^{+}\right)$and either $H^{+}=T^{+}\left(w, 2^{-(i+1)}\right)$ or $H^{+}=$ $\operatorname{int}\left(T^{+}\left(w, 2^{-(i+1)}\right)\right)$ for some $w \in \mathbb{R}^{2}$ (that lies directly above $x$ ) - in the first case, $H^{+} \subseteq T^{+}\left(x, 2^{-i}\right)$ for some $x \in A_{M, i}$, and in the second case, $H^{+}$is an open set with

$$
\nu\left(H^{+}\right) \leq \sup _{x \in A_{M, i} \cap T^{+}\left(z, 2^{-(i+1)}\right)} \nu\left(T^{+}\left(x, 2^{-i}\right)\right) \leq M 2^{-i(1+\xi)} .
$$

Since $\mathcal{L}\left(B_{M, i}\right) \leq 9$, we can use Fubini's theorem to estimate

$$
\begin{aligned}
9 M 2^{-i(1+\xi)} & \geq \int_{B_{M, i}} \nu\left(A_{M, i} \cap T^{+}\left(x, 2^{-(i+1)}\right)\right) d x \\
& =\int_{A_{M, i}} \mathcal{L}\left(B_{M, i} \cap T^{-}\left(y, 2^{-(i+1)}\right)\right) d \nu(y) \\
& \geq 2^{-(i+1)} \nu\left(A_{M, i}\right) .
\end{aligned}
$$

Hence

$$
\nu\left(A_{M, i}\right) \leq 18 M 2^{-i \xi},
$$

and so $\sum_{i} \nu\left(A_{M, i}\right)<\infty$, implying that

$$
\nu\left(\left\{x: \liminf _{i \rightarrow \infty} \frac{\nu\left(T^{+}\left(x, 2^{-i}\right)\right)}{2^{-i(1+\xi)}} \leq M\right\}\right)=0 .
$$

Since $\liminf _{r \rightarrow 0} r^{-(1+\xi)} \nu\left(T^{+}(x, r) \geq 2^{-(1+\xi)} \liminf _{i \rightarrow \infty} 2^{i(1+\xi)} \nu\left(T^{+}\left(x, 2^{-i}\right)\right)\right.$ for any $x \in \mathbb{R}^{2}$, the proposition follows. 


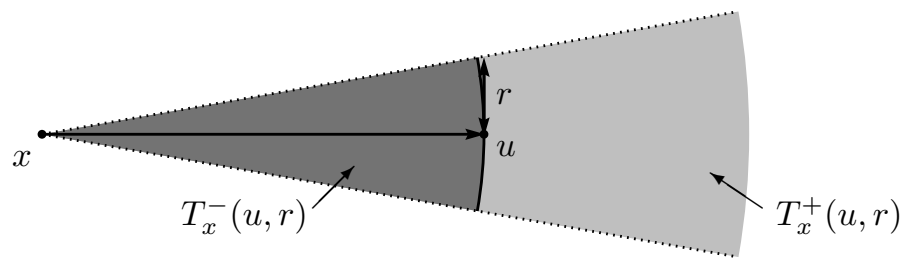

Figure 1 . The radial tubes $T_{x}^{-}(u, r)$ and $T_{x}^{+}(u, r)$

For $x \neq u \in \mathbb{R}^{2}$ and $r>0$, define radial tubes $T_{x}^{+}(u, r)$ and $T_{x}^{-}(u, r)$ by

$$
T_{x}^{+}(u, r)=V(x, u-x, r /|u-x|) \cap\left\{z \in \mathbb{R}^{2}:|z-x| \geq|u-x|\right\}
$$

and

$$
T_{x}^{-}(u, r)=V(x, u-x, r /|u-x|) \cap\left\{z \in \mathbb{R}^{2}:|z-x| \leq|u-x|\right\} ;
$$

see Figure 1 .

It is straightforward to transform our lemma about parallel tubes to one concerning radial tubes.

Lemma 2.6. Let $\nu$ be a compactly supported Radon measure in the plane and suppose $x \in \mathbb{R}^{2}$ satisfies $\nu(\{x\})=0$. Then for $\xi>0$ and for $\nu$-a.e. $u$,

$$
\liminf _{r \rightarrow 0} \frac{\nu\left(T_{x}^{+}(u, r)\right)}{r^{1+\xi}}=\liminf _{r \rightarrow 0} \frac{\nu\left(T_{x}^{-}(u, r)\right)}{r^{1+\xi}}=+\infty .
$$

Proof. Since $\nu(\{x\})=0$, it is enough to prove the lemma when there is a $\rho>0$ for which $\nu(B(x, \rho))=0$. Since $\operatorname{spt} \nu$ is compact, we can find some $R>\rho$ for which spt $\nu \subset B(x, R)$. Moreover, by restricting and translating $\nu$ suitably, we may suppose that $\operatorname{spt} \nu$ is a subset of a quadrant of the plane with corner at $x, Q(x)$ say, intersected with the annulus $A(x, \rho / 2, R)$. It is now straightforward to find an invertible transformation $T: Q(x) \cap A(x, \rho / 2, R) \rightarrow \mathbb{R}^{2}$ (namely, $r e^{i \theta} \mapsto(r, \theta)$ ) which transforms radial line segments from $x$ that intersect this region into halflines parallel to the $y$-axis. Moreover $\nu\left(T_{x}^{+}(u, r)\right)=\nu\left(T^{-1}\left(T^{+}(T(u), c r)\right)\right)$ for some constant $c>0$. This gives us the situation described in Proposition 2.5 for the image measure $T_{\sharp} \nu$, and the claim follows.

Later we shall use this lemma to show that for a measure with dimension greater than one, the radial tubes of a typical point $u, T_{x}^{ \pm}(u, r)$, have mass distributed relatively far from $u$ provided $r$ is sufficiently small. For now we just prove part of this statement. The set $E$ is introduced in the following lemma because there is no guarantee that the measure $\nu$ is supported on the right set when we use the lemma later in the paper.

Lemma 2.7. Let $s>1,0<r_{1} \leq r_{0} \leq 1$ and $\xi, M, d_{-}, c>0$, and $x \in \mathbb{R}^{2}$. Suppose that $\nu$ is a compactly supported Radon measure on the plane and that $F \subseteq E$ are compact sets in the plane satisfying:

(1) for all $u \in E,|u-x| \geq d_{-}$;

(2) for all $u \in E$ and $0<r \leq r_{0}$,

$$
\nu B(u, r) \leq c r^{s}
$$

(3) for all $u \in F$ and $0<r \leq r_{1}$,

$$
\nu\left(E \cap T_{x}^{ \pm}(u, r)\right)>M r^{1+\xi}
$$




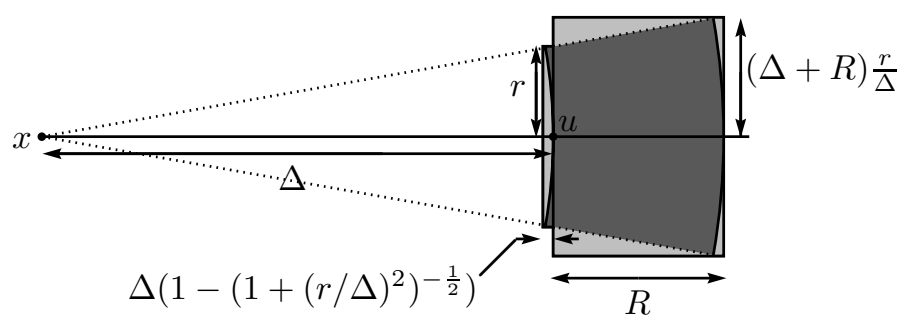

Figure 2. Estimating the mass of $T_{x}^{+}(u, r)$

Then there is $r_{2} \in\left(0,2^{-\frac{1}{2}} r_{1}\right]$ and $d_{0}>0$ such that for $u \in F$ and $0<r \leq r_{2}$,

$$
\nu\left(E \cap T_{x}^{ \pm}(u, r) \cap\left(\mathbb{R}^{2} \backslash A\left(x,|u-x|-d_{0} r^{2+\xi-s},|u-x|+d_{0} r^{2+\xi-s}\right)\right)\right)>0 .
$$

Proof. Let

$$
d_{0}=\frac{M}{13} 2^{-s / 2} \text { and } r_{2}=\min \left\{r_{1} / \sqrt{2}, \frac{1}{2} \sqrt{3} d_{-},\left(d_{-} / d_{0}\right)^{\frac{1}{2+\xi-s}}, d_{0}^{\frac{1}{s-1-\xi}}\right\} .
$$

We give the proof for $T_{x}^{+}(u, r)$; the proof for $T_{x}^{-}(u, r)$ is similar. By rotating and translating, we may assume that $x=0$ and that the line segment $[x, u]$ is on the positive $x$-axis. Let $\Delta=|u-x| \geq d_{-}$.

Elementary geometry shows, since $r \leq \frac{1}{2} \sqrt{3} d_{-} \leq \frac{1}{2} \sqrt{3} \Delta$ and so $\left(1+(r / \Delta)^{2}\right)^{-\frac{1}{2}} \geq$ $1-\frac{1}{2}(r / \Delta)^{2}$, that

$$
\begin{aligned}
& T_{x}^{+}(u, r) \cap A(x, \Delta-R, \Delta+R) \subseteq T_{x}^{+}(u, r) \cap B(x, \Delta+R) \\
& \subseteq\left(\left[\Delta-\frac{1}{2} r^{2} / \Delta, \Delta\right] \times[-r, r]\right) \cup([\Delta, \Delta+R] \times[-r(1+R / \Delta), r(1+R / \Delta)]),
\end{aligned}
$$

for any $R \geq 0$. We choose $R=d_{0} r^{2+\xi-s}$; see Figure 2 ,

We estimate that $T_{x}^{+}(u, r) \cap A(x, \Delta-R, \Delta+R)$ can be covered by

$$
2+(1+R / r) \times(2(1+R / \Delta)+1)
$$

closed squares of side $r$, since $\frac{1}{2} r^{2} / \Delta \leq \frac{1}{2} \frac{r_{2}}{d_{-}} r<r$. We find that

$$
2+(1+R / r) \times(2(1+R / \Delta)+1) \leq 2 R / r+(2 R / r)(2(1+1)+1) \leq 12 R / r,
$$

since $r \leq R \leq \Delta$ and $r \leq 1$. Hence we require at most $12 R / r$ balls of radius $\sqrt{2} r$ to cover $E \cap T_{x}^{+}(u, r) \cap(A, \Delta-R, \Delta+R)$.

So, since $\sqrt{2} r \leq r_{1} \leq r_{0}$, we estimate that

$$
\begin{aligned}
\nu\left(E \cap T_{x}^{+}(u, r) \cap(A, \Delta-R, \Delta+R)\right) \leq(12 R / r) \times 2^{s / 2} r^{s} & =12 \cdot 2^{\frac{s}{2}} d_{0} r^{1+\xi} \\
& <M r^{1+\xi},
\end{aligned}
$$

proving the lemma.

2.3. A 'two measures' estimate. In this subsection we investigate the interaction of two measures of dimension greater than one when they are supported on different visible sets of $\Gamma$. We show that if two measures of large dimension are supported in different visible sets, then they are 'disjoint' in the sense that balls containing points from both visible sets have small mass for both measures: this result is the key idea in our proof, and the remainder of the paper consists mainly of placing ourselves in a position to use it. 
In the following proposition, $\mathcal{T}(x, y, p)$ denotes the closed triangle with vertices $x, y$ and $p$, and $H(x, y ; u)$ denotes the closed upper half-plane that has the line segment $[x, y]$ in its boundary and $u$ lying in its interior.

Proposition 2.8. Let $\Gamma$ be a non-empty planar continuum. Suppose that $s>1$, $0<\xi<s-1,0<r_{1} \leq r_{0} \leq 1,0<d_{-} \leq d_{+}$and $M>0$ are given. Let $x, y \in \mathbb{R}^{2} \backslash \Gamma$ satisfy

$0<2|x-y|<d_{-} \leq \min \{d(x, \Gamma), d(y, \Gamma)\} \leq \max \{d(x, \Gamma), d(y, \Gamma)\}+\operatorname{diam}(\Gamma) \leq d_{+}$. Let $\nu_{x}$ and $\nu_{y}$ be Radon measures for which $\nu_{x}\left(\mathbb{R}^{2} \backslash \Gamma_{x}\right)=\nu_{y}\left(\mathbb{R}^{2} \backslash \Gamma_{y}\right)=0$, and let $E_{x}, F_{x}, E_{y}, F_{y}$ be compact sets for which

$$
F_{x} \subseteq E_{x} \subseteq \Gamma_{x} \text { and } F_{y} \subseteq E_{y} \subseteq \Gamma_{y}
$$

Suppose that:

(1) for all $u \in E_{x}, v \in E_{y}$ and $0<r \leq r_{0}$ both

$$
\nu_{x}\left(B(u, r) \leq r^{s} \text { and } \nu_{y}(B(v, r)) \leq r^{s} ;\right.
$$

(2) for all $u \in F_{x}, v \in F_{y}$ and $0<r \leq r_{1}$ both

$$
\nu_{x}\left(T_{x}^{ \pm}(u, r) \cap E_{x}\right) \geq M r^{1+\xi} \text { and } \nu_{y}\left(T_{y}^{ \pm}(v, r) \cap E_{y}\right) \geq M r^{1+\xi} ;
$$

(3) there is $\psi \in\left(0, \frac{1}{2}\right)$ such that for $u \in F_{x} \cup F_{y}$,

$$
\left\langle(u-x)^{\wedge},(u-y)^{\wedge}\right\rangle \in\left[\frac{1}{2}, 1-\psi\right] .
$$

Then there are constants $\alpha_{0}, d_{1}, c_{1}>0$ such that for $u \in F_{x}$, if $0<\rho \leq d_{1} \psi^{\frac{1}{2} \frac{1}{s-1-\xi}}$, then

$$
\nu_{y}\left(F_{y} \cap B(u, \rho)\right) \leq c_{1} \psi^{-\frac{1}{2}\left(\frac{s-1}{2+\xi-s}\right)} \rho^{\frac{1+\xi}{2+\xi-s}} .
$$

Furthermore, if $v \in F_{y} \cap B(u, \rho)$, then there is

$$
p \in\left[\frac{1}{2}(x+y), u\right] \cap B\left(u, \alpha_{0} \psi^{-1 / 2} \rho\right)
$$

such that $\mathcal{T}(x, y, p) \cap \Gamma=\emptyset$ and

$$
V\left(p, \frac{1}{2}(x+y)-u, \frac{2}{5} \psi^{\frac{1}{2}}\right) \cap \Gamma \cap H(x, y ; u)=\emptyset .
$$

Notice that the symmetry of the hypotheses in this proposition implies that a version of (2.1) holds for $u \in F_{y}$ with $\nu_{y}$ replaced by $\nu_{x}$ and $F_{y}$ replaced by $F_{x}$.

Proof. Let $\alpha_{0}=60 d_{+} / d_{-}, d_{1}=\min \left\{\left(r_{2} / \alpha_{1}\right)^{\frac{2+\xi-s}{s-1-\xi}}, d_{-} / \alpha_{0}, \frac{1}{20} d_{-}, r_{2}^{2+\xi-s}\right\}$ and $c_{1}=2^{5+s / 2} \alpha_{1}^{s-1}\left(d_{+} / d_{-}\right)$, where $\alpha_{1}^{2+\xi-s}=\left(\alpha_{0}+1\right) / d_{0}$, and $d_{0}$ and $r_{2}$ are the constants determined in Lemma 2.7. Suppose the conditions of the proposition are satisfied and let

we must show that

$$
0<\rho \leq d_{1} \psi^{\frac{1}{2} \frac{1}{s-1-\xi}}
$$

$$
\nu_{y}\left(F_{y} \cap B(u, \rho)\right) \leq c_{1} \psi^{-\frac{1}{2}\left(\frac{s-1}{2+\xi-s}\right)} \rho^{\frac{1+\xi}{2+\xi-s}} .
$$

If $F_{y} \cap B(u, \rho)=\emptyset$, then there is nothing to prove. So suppose $w \in F_{y} \cap B(u, \rho)$ and set (see Figure 3 )

$$
e=(u-x)^{\wedge}, \quad f=(w-y)^{\wedge} \text { and } g=(u-y)^{\wedge} .
$$

Notice that the third hypothesis of the proposition simply states that

$$
\frac{1}{2} \leq\langle e, g\rangle \leq 1-\psi
$$




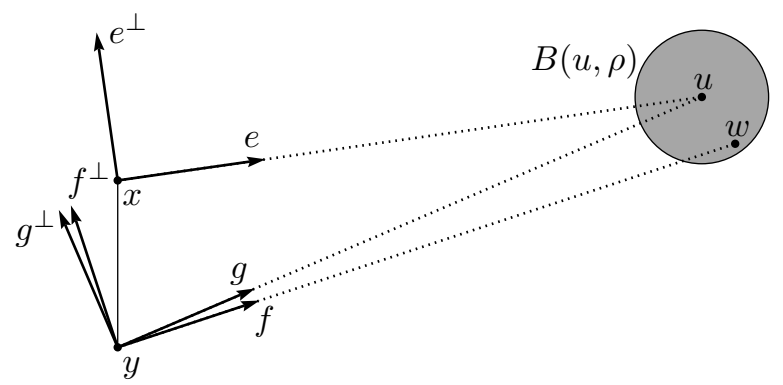

Figure 3. The vectors $e, f$ and $g$

and, since

$$
\left\langle e^{\perp}, g\right\rangle^{2}=1-\langle e, g\rangle^{2} \geq 1-(1-\psi)^{2}=\psi(2-\psi) \geq \psi,
$$

it follows that

$$
\left|\left\langle e^{\perp}, g\right\rangle\right| \geq \psi^{\frac{1}{2}} .
$$

We now make a sequence of geometric observations, of increasing complexity, about the relationships hypothesised in the proposition until we have enough information to prove the claims.

The first three observations are purely geometric and make no use of the measures $\nu_{x}$ and $\nu_{y}$.

In the first observation, we make some further simple estimates on the angles between various of the vectors $e, e^{\perp}, f, f^{\perp}, g$ and $g^{\perp}$.

Observation 1. If $0<\rho<\frac{1}{4} d_{-}$, then

$$
\begin{aligned}
\left|\left\langle f, g^{\perp}\right\rangle\right| & \leq \rho / d_{-}, \\
\langle f, g\rangle & \geq 1-2 \rho / d_{-}, \\
\langle e, f\rangle & >\frac{1}{4} d_{-} / d_{+} .
\end{aligned}
$$

Proof. For inequality (2.4), we use $g^{\perp}=\langle g, f\rangle f^{\perp}-\left\langle g, f^{\perp}\right\rangle f$ and calculate

$$
\left\langle f, g^{\perp}\right\rangle=0-\left\langle g, f^{\perp}\right\rangle=-\frac{1}{|u-y|}\left\langle u-y, f^{\perp}\right\rangle=-\frac{\left\langle u-w, f^{\perp}\right\rangle}{|u-y|} .
$$

Hence $\left|\left\langle f, g^{\perp}\right\rangle\right| \leq \rho / d_{-}$.

For inequality (2.5), on noting

$$
\langle f, g\rangle|w-y||u-y|=\langle w-y, u-y\rangle=\langle w-u, u-y\rangle+|u-y|^{2},
$$

we find

$$
\langle f, g\rangle=\frac{1}{|w-y|}\langle w-u, g\rangle+\frac{|u-y|}{|w-y|} \geq-\frac{\rho}{d_{-}}+\left(1-\frac{|u-w|}{|w-y|}\right) \geq 1-2 \rho / d_{-} .
$$

To verify inequality (2.6), note that $w=y+|w-y| f \in B(u, \rho)$, and so $w=$ $y+(x-y)+|u-x| e+z$ for some $|z| \leq \rho$. Hence $|w-y| f=(x-y)+|u-x| e+z$ and

$$
|w-y|\langle f, e\rangle=\langle x-y, e\rangle+|u-x|+\langle z, e\rangle .
$$

Now

$$
|\langle x-y, e\rangle| \leq \frac{1}{2} d_{-} \leq \frac{1}{2}|u-x| \quad \text { and } \quad|\langle z, e\rangle| \leq \rho \leq \frac{1}{4} d_{-} \leq \frac{1}{4}|u-x| .
$$


Thus

$$
|w-y|\langle e, f\rangle \geq \frac{1}{4}|u-x| \geq \frac{1}{4} d_{-}
$$

and so $\langle e, f\rangle \geq \frac{1}{4} d_{-} / d_{+}$, as required.

We now note that, since $u$ and $w$ are close, if $z \in V(y, w-y, r)$, then it is also in $V\left(y, u-y, r^{\prime}\right)$ for $r^{\prime}$ not too much larger than $r$.

Observation 2. If $0<\rho \leq \frac{1}{4} d_{-}$, then

$$
V\left(y, f, \rho / d_{-}\right) \subseteq V\left(y, g, 4 \rho / d_{-}\right) .
$$

Proof. If $z \in V\left(y, f, \rho / d_{-}\right)$, then

$$
\left|\left\langle z-y, f^{\perp}\right\rangle\right|<\frac{\rho}{d_{-}}\langle z-y, f\rangle .
$$

Since

$$
z-y=\langle z-y, f\rangle f+\left\langle z-y, f^{\perp}\right\rangle f^{\perp},
$$

we find

$$
\left\langle z-y, g^{\perp}\right\rangle=\langle z-y, f\rangle\left\langle f, g^{\perp}\right\rangle+\left\langle z-y, f^{\perp}\right\rangle\left\langle f^{\perp}, g^{\perp}\right\rangle .
$$

Hence (2.4) implies that

$$
\left|\left\langle z-y, g^{\perp}\right\rangle\right| \leq \frac{\rho}{d_{-}}|\langle z-y, f\rangle|+\left|\left\langle z-y, f^{\perp}\right\rangle\right| .
$$

Thus (2.7) gives

$$
\left|\left\langle z-y, g^{\perp}\right\rangle\right| \leq \frac{\rho}{d_{-}}|\langle z-y, f\rangle|+\frac{\rho}{d_{-}}\langle z-y, f\rangle=2\left(\rho / d_{-}\right)\langle z-y, f\rangle .
$$

It only remains to estimate $\langle z-y, f\rangle$ in terms of $\langle z-y, g\rangle$. Since $f=\langle f, g\rangle g+$ $\left\langle f, g^{\perp}\right\rangle g^{\perp}$,

$$
0<\langle z-y, f\rangle \leq\langle z-y, g\rangle\langle f, g\rangle+\left\langle z-y, g^{\perp}\right\rangle\left\langle f, g^{\perp}\right\rangle,
$$

which, on using (2.8) and (2.4), gives

$$
0<\langle z-y, f\rangle \leq\langle z-y, g\rangle\langle f, g\rangle+\frac{2 \rho}{d_{-}} \times \frac{\rho}{d_{-}}\langle z-y, f\rangle .
$$

Rearranging and using $0<\langle f, g\rangle \leq 1$, we find

$$
\langle z-y, f\rangle\left[1-2\left(\rho / d_{-}\right)^{2}\right] \leq\langle z-y, g\rangle \text {. }
$$

Substituting back into (2.8) then gives

$$
\left|\left\langle z-y, g^{\perp}\right\rangle\right| \leq 2\left(\rho / d_{-}\right)\left[1-2\left(\rho / d_{-}\right)^{2}\right]^{-1}\langle z-y, g\rangle,
$$

which, since $\rho \leq \frac{1}{2} d_{-}$, proves the claim.

The next observation is that the intersection of a cone from $x$ in the direction $u-x$ and a cone from $y$ in the direction $w-y$ is contained within a not too large ball centred on $u$ (or $w$ ) provided that the opening angle of the cones is sufficiently small.

Observation 3. If $0<\rho \leq \frac{1}{20} d_{-} \psi^{1 / 2}$, then

$$
V\left(x, e, \rho / d_{-}\right) \cap V\left(y, f, \rho / d_{-}\right) \subseteq B\left(u, \alpha_{0} \psi^{-1 / 2} \rho\right),
$$

where $\alpha_{0}=60 \frac{d_{+}}{d_{-}}$. 
Proof. Fix $z \in V\left(y, f, \rho / d_{-}\right) \cap V\left(x, e, \rho / d_{-}\right)$. Since $0<\rho \leq \frac{1}{20} d_{-} \psi^{1 / 2} \leq \frac{1}{4} d_{-}$, observation 2 implies that $z \in V\left(y, g, 4 \rho / d_{-}\right)$. Hence there are $\lambda, \mu>0$ for which

$$
z=y+\lambda\left(g-b g^{\perp}\right)=x+\mu\left(e+a e^{\perp}\right)
$$

where $|b| \leq 4 \rho / d_{-}$and $|a| \leq \rho / d_{-}$. We wish to find an upper bound for $|z-u|$. Notice that

$$
\langle z-x, e\rangle=\mu \quad \text { and } \quad\langle z-y, g\rangle=\lambda
$$

and

$$
|z-u|^{2}=\langle z-u, g\rangle^{2}+\left\langle z-u, g^{\perp}\right\rangle^{2}=(\lambda-|y-u|)^{2}+b^{2} \lambda^{2},
$$

and so upper estimates for $(\lambda-|y-u|)^{2}$ and $\lambda^{2}$ give an upper estimate for $|z-u|$.

Now

$$
\langle z-u, e\rangle=\langle y-u, e\rangle+\lambda\left(\langle g, e\rangle-b\left\langle g^{\perp}, e\right\rangle\right)=\langle x-u, e\rangle+\mu
$$

and so

$$
\mu=|x-u|-|u-y|\langle g, e\rangle+\lambda\left(\langle g, e\rangle-b\left\langle g^{\perp}, e\right\rangle\right) .
$$

However

$$
\left\langle z-u, e^{\perp}\right\rangle=\left\langle y-u, e^{\perp}\right\rangle+\lambda\left(\left\langle g, e^{\perp}\right\rangle-b\left\langle g^{\perp}, e^{\perp}\right\rangle\right)=a \mu,
$$

and so

$$
\begin{aligned}
& -|u-y|\left\langle g, e^{\perp}\right\rangle+\lambda\left(\left\langle g, e^{\perp}\right\rangle-b\left\langle g^{\perp}, e^{\perp}\right\rangle\right) \\
& =a|u-x|-a|u-y|\langle g, e\rangle+a \lambda\left(\langle g, e\rangle-b\left\langle g^{\perp}, e\right\rangle\right) .
\end{aligned}
$$

This rearranges to give

$$
\lambda \gamma=a|u-x|+|u-y|\left(\left\langle g, e^{\perp}\right\rangle-a\langle e, g\rangle\right),
$$

where $\gamma=(1-a b)\left\langle g, e^{\perp}\right\rangle-(a+b)\langle e, g\rangle$.

Since $|a| \leq \rho / d_{-}$and $|b| \leq 4 \rho / d_{-}$, it follows that $|a b| \leq \frac{1}{2}$ and $|a+b| \leq 5 \rho / d_{-}$. From equation (2.3) we know that $\left|\left\langle e^{\perp}, g\right\rangle\right| \geq \psi^{1 / 2}$, and so

$$
|\gamma| \geq \frac{1}{2} \psi^{1 / 2}-5\left(\rho / d_{-}\right) \geq \frac{1}{4} \psi^{1 / 2},
$$

since $\rho \leq \frac{1}{20} d_{-} \psi^{1 / 2}$.

Hence, since $|a| \leq \rho / d_{-} \leq 1$,

$$
|\lambda| \leq 4 \psi^{-1 / 2}\left[|a||u-x|+|u-y|\left(\left|\left\langle g, e^{\perp}\right\rangle\right|+|a||\langle e, g\rangle|\right)\right] \leq 12 d_{+} \psi^{-1 / 2} .
$$

Using (2.10), we find

$$
\begin{aligned}
\lambda-|u-y| & =\gamma^{-1}\left[a|u-x|+|u-y|\left(\left\langle g, e^{\perp}\right\rangle-a\langle e, g\rangle-\gamma\right)\right] \\
& =\gamma^{-1}\left[a|u-x|+b|u-y|\left(a\left\langle g, e^{\perp}\right\rangle+\langle e, g\rangle\right)\right],
\end{aligned}
$$

and so, since $|b| \leq 4 \rho / d_{-}$,

$$
\begin{aligned}
|\lambda-| u-y|| & \leq 4 \psi^{-1 / 2}\left[|a||u-x|+|b||u-y|\left(|a|\left|\left\langle g, e^{\perp}\right\rangle\right|+|\langle e, g\rangle|\right)\right] \\
& \leq 36\left(d_{+} / d_{-}\right) \psi^{-1 / 2} \rho .
\end{aligned}
$$

Thus (2.9) gives

$$
|z-u|^{2} \leq\left(36\left(d_{+} / d_{-}\right) \psi^{-1 / 2} \rho\right)^{2}+\left(48\left(d_{+} / d_{-}\right) \psi^{-1 / 2} \rho\right)^{2},
$$

and so

as claimed.

$$
|z-u| \leq 60\left(d_{+} / d_{-}\right) \psi^{-1 / 2} \rho
$$


We now show that there is a 'large' triangle with vertices at $x$ and $y$ and a third vertex close to $u$ such that the triangle is disjoint from $\Gamma$. This observation lies at the core of our entire argument and is the first time that we use the hypotheses involving the measures $\nu_{x}$ and $\nu_{y}$.

Observation 4. If $0<\rho \leq d_{1} \psi^{\frac{1}{2}\left(\frac{1}{s-1-\xi}\right)}$, and $r=\alpha_{1}\left(\psi^{-\frac{1}{2}} \rho\right)^{\frac{1}{2+\xi-s}}$, then there is

$$
z \in V\left(x, e, r / d_{-}\right) \cap V\left(y, f, r / d_{-}\right)
$$

with $\mathcal{T}(x, y, z) \cap \Gamma=\emptyset$ and $z \in B\left(u, \alpha_{0} \psi^{-1 / 2} \rho\right)$.

Proof. We aim to find a point $z$ that is visible from both $x$ and $y$. Recall that

$$
\alpha_{1}^{2+\xi-s}=\left(\alpha_{0}+1\right) / d_{0} \text { and } d_{1}=\min \left\{\left(r_{2} / \alpha_{1}\right)^{\frac{2+\xi-s}{s-1-\xi}}, d_{-} / \alpha_{0}, d_{-} / 20, r_{2}^{2+\xi-s}\right\} .
$$

(The constant $\alpha_{0}$ is as given in observation 3. and $r_{2}$ and $d_{0}$ are as given in Lemma 2.7.)

Since $w \in F_{y}$ and

$$
r=\alpha_{1}\left(\psi^{-\frac{1}{2}} \rho\right)^{\frac{1}{2+\xi-s}} \leq \alpha_{1} d_{1}^{\frac{1}{2+\xi-s}} \leq r_{2},
$$

we may use Lemma 2.7 applied to $\nu_{y}$ and $w$ to find $w^{\prime} \in E_{y} \cap T_{y}^{+}(w, r)$, and in particular lying in $V\left(y, w-y, r / d_{-}\right)$, for which

$$
\left|w-w^{\prime}\right|>d_{0} r^{2+\xi-s}=d_{0} \alpha_{1}^{2+\xi-s} \psi^{-\frac{1}{2}} \rho .
$$

Hence, since $|w-u| \leq \rho$,

$$
\left|w^{\prime}-u\right|>d_{0} \alpha_{1}^{2+\xi-s} \psi^{-\frac{1}{2}} \rho-\rho=\left(d_{0} \alpha_{1}^{2+\xi-s} \psi^{-\frac{1}{2}}-1\right) \rho \geq \alpha_{0} \psi^{-1 / 2} \rho .
$$

But

$$
\rho \leq d_{1} \psi^{\frac{1}{2}\left(\frac{1}{s-1-\xi}\right)} \leq d_{-} / \alpha_{0}<\frac{1}{4} d_{-}
$$

and

$$
r=\alpha_{1}\left(\psi^{-\frac{1}{2}} \rho\right)^{\frac{1}{2+\xi-s}} \rho^{-1} \rho=\alpha_{1}\left(\psi^{-\frac{1}{2}} \rho^{s-1-\xi}\right)^{\frac{1}{2+\xi-s}} \rho \leq \alpha_{1} d_{1}^{\frac{s-1-\xi}{2+\xi-s}} \rho \leq r_{2} \rho \leq \rho .
$$

Hence, by observation $2, w^{\prime} \in V\left(y, g, 4 \rho / d_{-}\right)$.

Similarly, there is $u^{\prime} \in E_{x} \cap T_{x}^{+}(u, r) \subseteq V\left(x, u-x, r / d_{-}\right)$for which

$$
\left|u^{\prime}-u\right| \geq \alpha_{0} \psi^{-1 / 2} \rho \text {; }
$$

see Figure 4.

Now both $|u-x|$ and $|u-y|$ are no less than $d_{-}$and

$$
\alpha_{0} \psi^{-1 / 2} \rho \leq \alpha_{0} d_{1} \psi^{\frac{1}{2}\left(\frac{2+\xi-s}{s-1-\xi}\right)}<\alpha_{0} d_{1} \leq d_{-} ;
$$

hence

$$
\min \{|u-x|,|u-y|\}>\alpha_{0} \psi^{-1 / 2} \rho
$$

Moreover

$$
r \leq \rho \leq d_{1} \psi^{\frac{1}{2}\left(\frac{1}{s-1-\xi}\right)} \leq d_{1} \psi^{\frac{1}{2}}<\frac{1}{20} d_{-} \psi^{1 / 2},
$$

and so it follows from observation 3 that

$$
\emptyset \neq\left[x, u^{\prime}\right] \cap\left[y, w^{\prime}\right] \subseteq B\left(u, \alpha_{0} \psi^{-1 / 2} \rho\right) .
$$




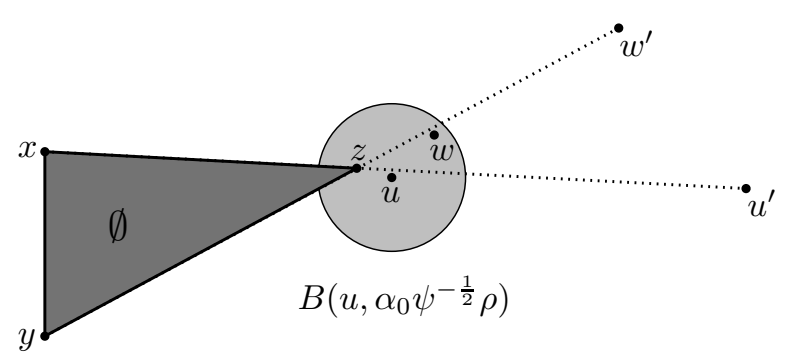

FigURE $4 . \emptyset \neq\left[x, u^{\prime}\right] \cap\left[y, w^{\prime}\right] \subseteq B\left(u, \alpha_{0} \psi^{-1 / 2} \rho\right)$

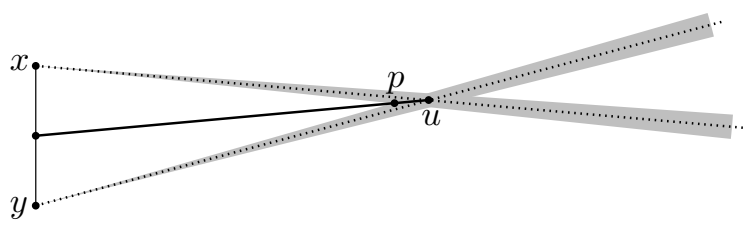

FigURE 5. $p \in\left[\frac{1}{2}(x+y), u\right] \cap\left(V\left(x, e, 4 \rho / d_{-}\right) \cup V\left(y, g, 4 \rho / d_{-}\right)\right)$

Let $z$ denote this intersection point. Then, since $u^{\prime}$ is visible from $x, w^{\prime}$ is visible from $y$ and $\Gamma$ is connected,

$$
([x, z] \cup[x, y] \cup[y, z]) \cap \Gamma=\emptyset .
$$

The observation follows.

We now refine this observation slightly and use it to find an empty cone with base point that is near to $u$.

Observation 5. If $0<\rho \leq d_{1} \psi^{\frac{1}{2}\left(\frac{1}{s-1-\xi}\right)}$, then there is $p \in\left[\frac{1}{2}(x+y), u\right] \cap$ $B\left(u, \alpha_{0} \psi^{-\frac{1}{2}} \rho\right)$ for which

$$
\mathcal{T}(x, y, p) \cap \Gamma=\emptyset \quad \text { and } \quad V\left(p, \frac{1}{2}(x+y)-u, \frac{2}{5} \psi^{1 / 2}\right) \cap H[x, y ; u] \cap \Gamma=\emptyset .
$$

Proof. Let

$$
p \in\left[\frac{1}{2}(x+y), u\right] \cap\left(\partial V\left(x, e, 4 \rho / d_{-}\right) \cup \partial V\left(y, g, 4 \rho / d_{-}\right)\right)
$$

be chosen to be at the minimum possible distance from $\frac{1}{2}(x+y)$; see Figure 5 . Then there is $\lambda>0$ such that

$$
p=u-\lambda \frac{|u-x| e+|u-y| g}{|| u-x|e+| u-y|g|} .
$$

We first show that $p \in B\left(u, \alpha_{0} \psi^{-\frac{1}{2}} \rho\right)$.

Suppose (without loss of generality) that $p \in V\left(y, g, 4 \rho / d_{-}\right)$. Then there exist $\mu>0$ and $\sigma \in\{+1,-1\}$ such that

$$
p=y+\mu\left(g+4 \rho d_{-}^{-1} \sigma g^{\perp}\right) .
$$

Hence, if we set $h=|u-x| e+|u-y| g$, then

$$
u-\lambda h /|h|=y+\mu\left(g+4 \rho d_{-}^{-1} \sigma g^{\perp}\right),
$$


which, since $u-y=|u-y| g$, rearranges to give

$$
|u-y| g-\lambda h /|h|=\mu\left(g+4 \rho d_{-}^{-1} \sigma g^{\perp}\right) .
$$

So taking the inner product of (2.11) with $g^{\perp}$ gives

$$
-\lambda \frac{|u-x|}{|h|}\left\langle e, g^{\perp}\right\rangle=\frac{4 \rho}{d_{-}} \sigma \mu,
$$

and taking the inner product of (2.11) with $g$ and rearranging gives

$$
|u-y|-\lambda \frac{|u-x|\langle e, g\rangle+|u-y|}{|h|}=\mu .
$$

Substituting for $\mu$ from (2.12) gives

$$
|u-y|-\lambda \frac{|u-x|\langle e, g\rangle+|u-y|}{|h|}=-\frac{d_{-}}{4 \rho \sigma} \cdot \frac{|u-x|\left\langle e, g^{\perp}\right\rangle}{|h|} \lambda
$$

and this rearranges to give

$$
\lambda\left[\left(\frac{d_{-}}{4 \rho \sigma}\left\langle e, g^{\perp}\right\rangle-\langle e, g\rangle\right)|u-x|-|u-y|\right]=-|u-y||h|
$$

and so, substituting for $h$,

$$
\lambda\left[\left(d_{-}\left\langle e, g^{\perp}\right\rangle-4 \rho \sigma\langle e, g\rangle\right)-4 \rho \sigma \frac{|u-y|}{|u-x|}\right]=-4 \rho \sigma|u-y|\left|e+\frac{|u-y|}{|u-x|} g\right| .
$$

Since $|x-y| \leq \frac{1}{2} d_{-}$, it easily follows that

$$
\frac{2}{3} \leq \frac{|u-x|}{|u-y|}, \frac{|u-y|}{|u-x|} \leq \frac{3}{2},
$$

and so

$$
|\lambda| \leq \frac{4 \rho}{d_{-}} \times\left(1+\frac{3}{2}\right) \frac{|u-y|}{\left|\sigma\left\langle e, g^{\perp}\right\rangle-4 \rho d_{-}^{-1}(\langle e, g\rangle+|u-y| /|u-x|)\right|} .
$$

Now $|\langle e, g\rangle+| u-y|/| u-x|| \leq \frac{5}{2}$ and by (2.3),

$$
\left|\left\langle e, g^{\perp}\right\rangle\right|=\left|\left\langle e^{\perp}, g\right\rangle\right| \geq \psi^{1 / 2} .
$$

Thus, since $\rho \leq d_{1} \psi^{\frac{1}{2}\left(\frac{1}{s-1-\xi}\right)} \leq d_{1} \psi^{\frac{1}{2}}<\frac{1}{20} d_{-} \psi^{1 / 2}$,

$$
\left|\sigma\left\langle e, g^{\perp}\right\rangle-4 \rho d_{-}^{-1}(\langle e, g\rangle+|u-y| /|u-x|)\right| \geq \psi^{1 / 2}-10 \rho / d_{-} \geq \frac{1}{2} \psi^{1 / 2} .
$$

Hence

$$
|\lambda| \leq 10 \rho d_{-}^{-1}|u-y| \times 2 \psi^{-1 / 2} \leq 20\left(d_{+} / d_{-}\right) \psi^{-1 / 2} \rho \leq \alpha_{0} \psi^{-\frac{1}{2}} \rho,
$$

and $p \in B\left(u, \alpha_{0} \psi^{-1 / 2} \rho\right)$, as claimed.

Since the hypotheses of observation 4 are satisfied, there is a point $z$ satisfying its conclusions, and we note that $p \in \mathcal{T}(x, y, z)$. Hence $\mathcal{T}(x, y, p) \cap \Gamma=\emptyset$.

To show that

$$
V\left(p, \frac{1}{2}(x+y)-u, \frac{2}{5} \psi^{1 / 2}\right) \cap H[x, y ; u] \cap \Gamma=\emptyset,
$$

we recall that $h=|u-x| e+|u-y| g$ and so

$$
h^{\perp}=e^{\perp}|u-x|+g^{\perp}|u-y| .
$$

We shall estimate $\left|\left\langle x-u, h^{\perp}\right\rangle\right|,\left|\left\langle y-u, h^{\perp}\right\rangle\right|,|\langle x-u, h\rangle|$ and $|\langle y-u, h\rangle|$; for if $q \in H(x, y ; u)$ satisfies

$$
\left|\left\langle q-p, h^{\perp}\right\rangle\right| \leq m\langle q-p, h\rangle
$$


where $m=\min \left\{\left|\left\langle x-u, h^{\perp}\right\rangle /\langle x-u, h\rangle\right|,\left|\left\langle y-u, h^{\perp}\right\rangle /\langle y-u, h\rangle\right|\right\}$, then $q \in$ $\mathcal{T}(x, y, p)$. Now

$$
\begin{aligned}
\left\langle x-u, h^{\perp}\right\rangle & =-|x-u||y-u|\left\langle e, g^{\perp}\right\rangle \\
\langle x-u, h\rangle & =-|x-u|^{2}-|x-u||y-u|\langle e, g\rangle \\
\left\langle y-u, h^{\perp}\right\rangle & =-|x-u||y-u|\left\langle g, e^{\perp}\right\rangle \\
\langle y-u, h\rangle & =-|y-u|^{2}-|x-u||y-u|\langle e, g\rangle,
\end{aligned}
$$

and so

$$
\left|\frac{\left\langle x-u, h^{\perp}\right\rangle}{\langle x-u, h\rangle}\right|=\left|\frac{\left\langle e, g^{\perp}\right\rangle}{\langle e, g\rangle+|x-u| /|y-u|}\right|
$$

and

$$
\left|\frac{\left\langle y-u, h^{\perp}\right\rangle}{\langle y-u, h\rangle}\right|=\left|\frac{\left\langle e, g^{\perp}\right\rangle}{\langle e, g\rangle+|y-u| /|x-u|}\right| .
$$

Hence $m \geq \frac{2}{5} \psi^{1 / 2}$ and the observation follows.

The existence of a large empty cone near to $u$ and $w$ forces all other points of $F_{y} \cap B(u, \rho)$ to lie in a relatively narrow cone in the direction $w-y$.

Observation 6. Let $0<\rho \leq d_{1} \psi^{\frac{1}{2}\left(\frac{1}{s-1-\xi}\right)}$ and $r=\alpha_{1}\left(\psi^{-\frac{1}{2}} \rho\right)^{\frac{1}{2+\xi-s}}$. If $v, w \in$ $F_{y} \cap B(u, \rho)$, then $v \in V\left(y, w-y, 3 r / d_{-}\right)$.

Proof. Suppose that $\left\langle v-y,(w-y)^{\perp}\right\rangle>0$. (If not, then interchange the labelling of $v$ and $w$ - notice that $\left\langle v-y,(w-y)^{\perp}\right\rangle \neq 0$ since $v$ and $w$ are both visible from y.) By observation 4 , there exists

$$
z \in V\left(x, e, r / d_{-}\right) \cap V\left(y, f, r / d_{-}\right) \cap B\left(u, \alpha_{0} \psi^{-1 / 2} \rho\right)
$$

for which $\Gamma \cap \mathcal{T}(x, y, z)=\emptyset$.

By Lemma 2.7 we can find $v^{\prime} \in E_{y} \cap T_{y}^{-}(v, r)$ for which

$$
\left|v^{\prime}-u\right| \geq\left|v^{\prime}-v\right|-|u-v|>d_{0} r^{2+\xi-s}-\rho=\left(d_{0} \alpha_{1}^{2+\xi-s} \psi^{-\frac{1}{2}}-1\right) \rho=\alpha_{0} \psi^{-1 / 2} \rho .
$$

We show that if

$$
\left\langle v-y,(w-y)^{\perp}\right\rangle \geq 3\left(r / d_{-}\right)\langle v-y, w-y\rangle,
$$

then $v^{\prime} \in \mathcal{T}(x, y, z)$, contradicting $\mathcal{T}(x, y, z) \cap \Gamma=\emptyset$. To do this, since $z \in$ $V\left(x, e, r / d_{-}\right) \cap V\left(y, f, r / d_{-}\right)$, it is enough to show that

$$
\left\langle v^{\prime}-x, e^{\perp}\right\rangle<-\left(r / d_{-}\right)\left\langle v^{\prime}-x, e\right\rangle
$$

and

$$
\left\langle v^{\prime}-y,(w-y)^{\perp}\right\rangle>\left(r / d_{-}\right)\left\langle v^{\prime}-y, w-y\right\rangle .
$$

To show (2.15): If

$$
v^{\prime} \in V\left(y, v-y, r / d_{-}\right) \cap V\left(x, e, r / d_{-}\right),
$$

then observation 3 applied to $v$ implies that $\left|v^{\prime}-u\right|<\alpha_{0} \psi^{-1 / 2} \rho$, contradicting (2.13). Hence, since $v^{\prime} \in V\left(y, v-y, r / d_{-}\right)$, we deduce

$$
\left|\left\langle v^{\prime}-x, e^{\perp}\right\rangle\right|>\left(r / d_{-}\right)\left\langle v^{\prime}-x, e\right\rangle,
$$


and it only remains to show that $\left\langle v^{\prime}-x, e^{\perp}\right\rangle\left\langle 0\right.$. If $\left\langle v^{\prime}-x, e^{\perp}\right\rangle \geq 0$, then $\left\langle v^{\prime}-x, e^{\perp}\right\rangle>\left(r / d_{-}\right)\left\langle v^{\prime}-x, e\right\rangle$. But, by observation 2 applied to $v,\left|\left\langle v^{\prime}-y, g^{\perp}\right\rangle\right|$ $<4\left(\rho / d_{-}\right)\left\langle v^{\prime}-y, g\right\rangle$ and so, since $\left|v^{\prime}-y\right|<|v-y|$, we find

$$
\begin{aligned}
\left|\left\langle v^{\prime}-y, g^{\perp}\right\rangle\right|<4\left(\rho / d_{-}\right)|v-y| & \leq 4\left(\rho / d_{-}\right)(|u-y|+\rho) \\
& \leq\left(5 \rho / d_{-}\right)|u-y|, \text { since } \rho<\frac{1}{4} d_{-} \\
& \leq 5\left(d_{+} / d_{-}\right) \rho .
\end{aligned}
$$

But $\left\langle v^{\prime}-u, g^{\perp}\right\rangle=\left\langle v^{\prime}-y, g^{\perp}\right\rangle$, and so

$$
\left|\left\langle v^{\prime}-u, g^{\perp}\right\rangle\right| \leq 5\left(d_{+} / d_{-}\right) \rho .
$$

Now

$$
\left\langle v^{\prime}-u, g\right\rangle=\left\langle v^{\prime}-y, g\right\rangle-|u-y| \leq|v-y|-|u-y| \leq \rho .
$$

Let $q$ be the point of intersection of $[x, u]$ with $\left[y, y+|y-u|\left(g+4\left(\rho / d_{-}\right) g^{\perp}\right)\right]$. Then, since $v^{\prime} \in V\left(y, g, 4\left(\rho / d_{-}\right)\right)$and $\left\langle v^{\prime}-x, e^{\perp}\right\rangle>\left(r / d_{-}\right)\left\langle v^{\prime}-x, e\right\rangle$, it follows that $\left\langle v^{\prime}-u, g\right\rangle \geq\langle q-u, g\rangle$. Hence it is enough to find a lower bound for $\langle q-u, g\rangle$. There exist $0<\lambda<|u-y|$ and $0<\mu<|x-u|$ such that

$$
q=y+\lambda\left(g+4\left(\rho / d_{-}\right) g^{\perp}\right)=x+\mu e,
$$

and so

$$
-|u-y| g+\lambda\left(g+4\left(\rho / d_{-}\right) g^{\perp}\right)=(\mu-|u-x|) e .
$$

Taking inner products of this expression with $g$ and $g^{\perp}$, and solving for $\lambda$, gives

$$
\lambda=|u-y|\left(1+4\left(\frac{\rho}{d_{-}}\right) \frac{\langle e, g\rangle}{\left\langle e^{\perp}, g\right\rangle}\right)^{-1} \text {. }
$$

Hence

$$
\lambda \geq|u-y|\left(1+4\left(\rho / d_{-}\right) \psi^{-1 / 2}\right)^{-1} \geq|u-y|\left(1-4\left(\rho / d_{-}\right) \psi^{-1 / 2}\right) .
$$

Thus

and so

$$
\langle q-u, g\rangle \geq-4\left(\rho / d_{-}\right) \psi^{-1 / 2}|u-y|
$$

$$
\left\langle v^{\prime}-u, g\right\rangle \geq-4\left(\rho / d_{-}\right) \psi^{-1 / 2}|u-y| \geq-4\left(d_{+} / d_{-}\right) \psi^{-\frac{1}{2}} \rho
$$

Hence

$$
\left|v^{\prime}-u\right| \leq 5 \rho \frac{d_{+}}{d_{-}}+4 \rho \psi^{-1 / 2} \frac{d_{+}}{d_{-}} \leq 9\left(d_{+} / d_{-}\right) \psi^{-1 / 2} \rho<\alpha_{0} \psi^{-1 / 2} \rho,
$$

contradicting (2.13).

For (2.16), notice that $v^{\prime}-y=\alpha(v-y)+\beta(v-y)^{\perp}$ for some $0<\alpha<1$ and $|\beta|<\alpha\left(r / d_{-}\right)$. Thus, using (2.14),

$$
\begin{aligned}
\left\langle v^{\prime}-y,(w-y)^{\perp}\right\rangle & =\alpha\left\langle v-y,(w-y)^{\perp}\right\rangle+\beta\left\langle(v-y)^{\perp},(w-y)^{\perp}\right\rangle \\
& \geq 3 \alpha\left(r / d_{-}\right)\langle v-y, w-y\rangle-|\beta||\langle v-y, w-y\rangle| \\
& \geq 2 \alpha r / d_{-}\langle v-y, w-y\rangle
\end{aligned}
$$

and

$$
\left\langle v^{\prime}-y, w-y\right\rangle=\alpha\langle v-y, w-y\rangle+\beta\left\langle(v-y)^{\perp}, w-y\right\rangle
$$

But

$$
\left\langle(v-y)^{\perp}, w-y\right\rangle=\left\langle(v-y)^{\perp}, w-v\right\rangle
$$

and so $\left|\left\langle(v-y)^{\perp}, w-y\right\rangle\right| \leq 2 \rho|v-y|$, and

$$
\langle v-y, w-y\rangle=\langle v-y, w-v\rangle+|v-y|^{2} .
$$


Hence

$$
|\langle v-y, w-y\rangle| \geq|v-y|(|v-y|-2 \rho) \geq \frac{1}{2} d_{-}|v-y|
$$

since $|v-y| \geq d_{-}$and $\rho \leq \frac{1}{4} d_{-}$. Thus

$$
\left|\left\langle(v-y)^{\perp}, w-y\right\rangle\right| \leq 2 \rho|v-y| \leq 4\left(\rho / d_{-}\right)\langle v-y, w-y\rangle .
$$

So

$$
\begin{aligned}
\left\langle v^{\prime}-y, w-y\right\rangle & \leq\left(\alpha+4\left(\rho / d_{-}\right)|\beta|\right)\langle v-y, w-y\rangle \\
& \leq\left(1+4\left(\rho / d_{-}\right)\left(r / d_{-}\right)\right) \alpha\langle v-y, w-y\rangle \\
& <\left(1+4\left(\rho / d_{-}\right)\left(r / d_{-}\right)\right)\left(d_{-} /(2 r)\right)\left\langle v^{\prime}-y,(w-y)^{\perp}\right\rangle
\end{aligned}
$$

and rearranging gives

$$
\left\langle v^{\prime}-y,(w-y)^{\perp}\right\rangle>\left(2 r / d_{-}\right)\left(1+4\left(\rho / d_{-}\right)\left(r / d_{-}\right)\right)^{-1}\left\langle v^{\prime}-y, w-y\right\rangle,
$$

which, since $4\left(\rho / d_{-}\right)\left(r / d_{-}\right)<r / d_{-} \leq r_{2} / d_{-}<1$, implies (2.16).

We can now finish the proof of the proposition. Then

Let $0<\rho \leq d_{1} \psi^{\frac{1}{2}\left(\frac{1}{s-1-\xi}\right)}$ and $r=\alpha_{1}\left(\psi^{-\frac{1}{2}} \rho\right)^{\frac{1}{2+\xi-s}}$. Suppose $w \in B(u, \rho) \cap F_{y}$.

$$
F_{y} \cap B(u, \rho) \subset V\left(y,(w-y), 3 r / d_{-}\right) .
$$

Thus $F_{y} \cap B(u, \rho)$ is contained in a rectangle of height $2 \rho$ and width $6 r d_{+} / d_{-}$which can be covered by $(2+2 \rho / r)\left(2+6 d_{+} / d_{-}\right)$boxes of side $r$. Since

$$
(2+2 \rho / r)\left(2+6 d_{+} / d_{-}\right) \leq 32(\rho / r) d_{+} / d_{-},
$$

and $\sqrt{2} r \leq \sqrt{2} \alpha_{1}\left(d_{1} \psi^{\frac{1}{2}\left(\frac{2+\xi-s}{s-1-\xi}\right)}\right)^{\frac{1}{2+\xi-s}} \leq \sqrt{2} \alpha_{1} d_{1}^{\frac{1}{2+\xi-s}} \leq \sqrt{2} r_{2} \leq r_{0}$, we estimate that

$$
\nu_{y}\left(F_{y} \cap B(u, \rho)\right) \leq 2^{5+s / 2}\left(d_{+} / d_{-}\right) \rho r^{s-1}=2^{5+s / 2} \alpha_{1}^{s-1}\left(d_{+} / d_{-}\right) \psi^{-\frac{1}{2}\left(\frac{s-1}{2+\xi-s}\right)} \rho^{\frac{1+\xi}{2+\xi-s}},
$$

as required. The remainder of the proposition follows from observation 5 .

2.4. A mixed mass estimate. The main utility of Proposition 2.8 lies in its use in proving the following proposition, where we obtain an estimate of the 'mixed mass' $\nu_{x} \otimes \nu_{y}(A \times B)$ for appropriate planar sets $A$ and $B$. (Here $\nu_{x} \otimes \nu_{y}$ is the product measure of $\nu_{x}$ and $\nu_{y}$.)

Proposition 2.9. Let $\Gamma$ be a non-empty planar continuum. Suppose that $s>1$, $0<\xi<s-1,0<r_{1} \leq r_{0} \leq 1,0<d_{-} \leq d_{+}$and $M>0$ are given. Let $x, y \in \mathbb{R}^{2} \backslash \Gamma$ satisfy

$0<2|x-y|<d_{-} \leq \min \{d(x, \Gamma), d(y, \Gamma)\} \leq \max \{d(x, \Gamma), d(y, \Gamma)\}+\operatorname{diam}(\Gamma) \leq d_{+}$.

Let $\nu_{x}$ and $\nu_{y}$ be Radon measures supported in $\Gamma_{x}$ and $\Gamma_{y}$, respectively, and suppose that $F_{x}, E_{x}, F_{y}$ and $E_{y}$ are compact sets for which

$$
F_{x} \subseteq E_{x} \subseteq \Gamma_{x} \text { and } F_{y} \subseteq E_{y} \subseteq \Gamma_{y} .
$$

Suppose that:

(1) for all $u \in E_{x}, v \in E_{y}$ and $0<r \leq r_{0}$, both

$$
\nu_{x}\left(B(u, r) \leq r^{s} \text { and } \nu_{y}(B(v, r)) \leq r^{s} ;\right.
$$

(2) for all $u \in F_{x}, v \in F_{y}$ and $0<r \leq r_{1}$, both

$$
\nu_{x}\left(T_{x}^{ \pm}(u, r) \cap E_{x}\right) \geq M r^{1+\xi} \text { and } \nu_{y}\left(T_{y}^{ \pm}(v, r) \cap E_{y}\right) \geq M r^{1+\xi} ;
$$


(3) there exists $\psi \in\left(0, \frac{1}{2}\right)$ such that for $u \in F_{x} \cup F_{y}$,

$$
\left\langle(u-x)^{\wedge},(u-y)^{\wedge}\right\rangle \in\left[\frac{1}{2}, 1-\psi\right] .
$$

Then there are constants $c_{2}, d_{2}>0$ such that if $0<\rho \leq d_{2} \psi^{\frac{1}{2} \frac{1}{s-1-\xi}}$, then

$$
\begin{aligned}
\left(\nu_{x} \otimes \nu_{y}\right)\left(\left(F_{x} \times F_{y}\right)\right. & \cap\{(u, v):|u-v| \leq \rho\}) \\
\leq & c_{2} \operatorname{arc-diam}_{\frac{1}{2}(x+y)}\left(F_{x} \cap B\left(F_{y}, \rho\right)\right)\left(\psi^{-\frac{1}{2}} \rho\right)^{\frac{s+\xi}{2+\xi-s}} .
\end{aligned}
$$

Proof. Using the constants established in Proposition 2.8, let $d_{2}=\frac{5}{2^{3 / 2} \alpha_{0}} d_{1}$. Observe that, since $d_{2} \leq d_{1}$, Proposition 2.8 implies that

$$
\begin{aligned}
& \left(\nu_{x} \otimes \nu_{y}\right)\left(\left(F_{x} \times F_{y}\right) \cap\{(u, v):|u-v| \leq \rho\}\right) \\
& =\left.\left.\int_{B\left(F_{y}, \rho\right)} \nu_{y}\right|_{F_{y}}(\{v:|u-v| \leq \rho\}) d \nu_{x}\right|_{F_{x}}(u) \\
& =\left.\int_{B\left(F_{y}, \rho\right)} \nu_{y}\left(F_{y} \cap B(u, \rho)\right) d \nu_{x}\right|_{F_{x}}(u) \\
& \leq c_{1} \psi^{-\frac{s-1}{2(2+\xi-s)}} \rho^{\frac{1+\xi}{2+\xi-s}} \nu_{x}\left(F_{x} \cap B\left(F_{y}, \rho\right)\right) .
\end{aligned}
$$

It remains to estimate

$$
\nu_{x}\left(F_{x} \cap B\left(F_{y}, \rho\right)\right) .
$$

We begin by noticing that for each

$$
u \in F_{x} \cap B\left(F_{y}, \rho\right),
$$

Proposition 2.8 guarantees the existence of a $p_{u} \in\left[\frac{1}{2}(x+y), u\right] \cap B\left(u, \alpha_{0} \psi^{-1 / 2} \rho\right)$ for which $\mathcal{T}\left(x, y, p_{u}\right) \cap \Gamma=\emptyset$ and

$$
V\left(p_{u}, \frac{1}{2}(x+y)-u, \frac{2}{5} \psi^{\frac{1}{2}}\right) \cap \Gamma \cap H(x, y ; u)=\emptyset .
$$

Let $\sigma=\frac{2}{5} \frac{\alpha_{0}}{d_{+}} \rho$ and fix $v \in F_{x} \cap B\left(F_{y}, \rho\right)$. Then Lemma 2.2 guarantees that if

$$
w \in V\left(\frac{1}{2}(x+y), p_{v}-\frac{1}{2}(x+y), \sigma\right) \backslash V\left(p_{v},-\left(p_{v}-\frac{1}{2}(x+y)\right), \frac{2}{5} \psi^{\frac{1}{2}}\right),
$$

then

$$
\begin{aligned}
\left\langle w-p_{v},\left(p_{v}-\frac{1}{2}(x+y)\right)^{\wedge}\right\rangle & \geq-\frac{\sigma}{\sigma+\frac{2}{5} \psi^{\frac{1}{2}}}\left|p_{v}-\frac{1}{2}(x+y)\right| \\
& \geq-\frac{5}{2} d_{+}\left(\frac{2}{5} \frac{\alpha_{0}}{d_{+}}\right) \psi^{-\frac{1}{2}} \rho=-\alpha_{0} \psi^{-\frac{1}{2}} \rho .
\end{aligned}
$$

So suppose $u, v \in F_{x}$ with $u \in V\left(\frac{1}{2}(x+y), v, \sigma\right)$ and assume, without loss of generality, that

$$
\left|u-\frac{1}{2}(x+y)\right| \leq\left|v-\frac{1}{2}(x+y)\right| .
$$

We wish to estimate $\left\langle u-v,\left(v-\frac{1}{2}(x+y)\right)^{\wedge}\right\rangle$ from below. (An easy upper bound is given by zero.) From the preceding we know that

$$
\left\langle u-p_{v},\left(v-\frac{1}{2}(x+y)\right)^{\wedge}\right\rangle=\left\langle u-p_{v},\left(p_{v}-\frac{1}{2}(x+y)\right)^{\wedge}\right\rangle \geq-\alpha_{0} \psi^{-\frac{1}{2}} \rho .
$$

Hence

$$
\begin{aligned}
\left\langle u-v,\left(v-\frac{1}{2}(x+y)\right)^{\wedge}\right\rangle & =\left\langle u-p_{v},\left(v-\frac{1}{2}(x+y)\right)^{\wedge}\right\rangle+\left\langle p_{v}-v,\left(v-\frac{1}{2}(x+y)\right)^{\wedge}\right\rangle \\
& \geq-\alpha_{0} \psi^{-\frac{1}{2}} \rho-\alpha_{0} \psi^{-\frac{1}{2}} \rho \\
& \geq-2 \alpha_{0} \psi^{-\frac{1}{2}} \rho .
\end{aligned}
$$


Thus

$$
V\left(\frac{1}{2}(x+y), v-\frac{1}{2}(x+y), \sigma\right) \cap\left(F_{x} \cap B\left(F_{y}, \rho\right) \cap B\left(\frac{1}{2}(x+y),\left|v-\frac{1}{2}(x+y)\right|\right)\right)
$$

can be covered by $\left(2 \alpha_{0} \psi^{-1 / 2} \rho\right) /\left(\frac{2}{5} \alpha_{0} \rho\right)=5 \psi^{-\frac{1}{2}}$ boxes of side $\frac{4}{5} \alpha_{0} \rho$. Hence, by using the mass estimate in Proposition 2.8 (for $\nu_{x}$ rather than $\nu_{y}$ ), since $2 \sqrt{2} \frac{2}{5} \alpha_{0} \rho \leq$ $d_{1} \psi^{\frac{1}{2} \frac{1}{s-1-\xi}}$

$$
\nu_{x}\left(V\left(\frac{1}{2}(x+y), v-\frac{1}{2}(x+y), \sigma\right) \cap F_{x} \cap B\left(F_{y}, \rho\right) \cap B\left(\frac{1}{2}(x+y),\left|v-\frac{1}{2}(x+y)\right|\right)\right)
$$

is at most

$$
5 \psi^{-\frac{1}{2}} \times c_{1} \psi^{-\frac{1}{2} \frac{s-1}{2+\xi-s}}\left(\frac{4 \sqrt{2}}{5} \alpha_{0} \rho\right)^{\frac{1+\xi}{2+\xi-s}}=5 c_{1}\left(\frac{4 \sqrt{2}}{5} \alpha_{0}\right)^{\frac{1+\xi}{2+\xi-s}}\left(\psi^{-\frac{1}{2}} \rho\right)^{\frac{1+\xi}{2+\xi-s}} .
$$

By choosing $v$ to be as far from $\frac{1}{2}(x+y)$ as possible and counting the number of such cones needed to cover $F_{x} \cap A$, we obtain

$$
\begin{aligned}
& \nu_{x}\left(F_{x} \cap B\left(F_{y}, \rho\right)\right)
\end{aligned}
$$

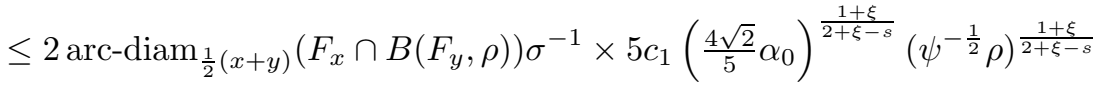

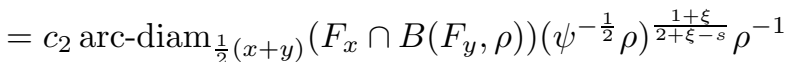

for $c_{2}=25 c_{1} d_{+}(4 \sqrt{2} / 5)^{\frac{1+\xi}{2+\xi-s}} \alpha_{0}^{\frac{s-1}{2+\xi-s}}$, and this implies the claim.

\section{Measurability Results}

The aim in this section is to prove the following proposition that encapsulates the standard uniformisation procedures we require. It allows us to find sets and measures where the phenomena that we are interested in occur in a controlled and measurable way. The results of this section do not require that $\Gamma$ is connected.

Proposition 3.1. Let $\Gamma$ be a non-empty compact subset of the plane. Let $C$ be a closed ball disjoint from $\Gamma$ and $U$ an open set.

Suppose that $s \in(1,2)$ and $t \in(0,2)$ are such that

$$
\operatorname{dim}_{H}\left(\left\{x \in C: \operatorname{dim}_{H}\left(\Gamma_{x} \cap U\right)>s\right\}\right)>t .
$$

Then for each $\xi \in(0, s-1)$, we can find $M, p, q, c>0$, a compact set $D \subseteq C$ and a Radon measure $\mu$ with $0<\mu(D) \leq 1$ such that:

(1) for each $x \in D$ and $r>0, \mu(B(x, r)) \leq r^{t}$;

(2) for each $x \in D$, there is a Radon measure $\nu_{x}$ and compact sets $F_{x} \subseteq E_{x}$ contained in $U \cap \Gamma_{x}$ such that:

(a) $\nu_{x}\left(\mathbb{R}^{2} \backslash \Gamma_{x}\right)=0, \nu_{x}\left(\mathbb{R}^{2}\right) \leq c$ and for $0<r \leq 1$ and $u \in \Gamma, \nu_{x}(B(u, r)) \leq$ $r^{s}$

(b) $\nu_{x}\left(F_{x}\right)>p$

(c) for $0<r \leq q$ and $u \in F_{x}$,

$$
\min \left\{\nu_{x}\left(T_{x}^{+}(u, r) \cap E_{x}\right), \nu_{x}\left(T_{x}^{-}(u, r) \cap E_{x}\right)\right\}>M r^{1+\xi} .
$$

Moreover $\nu$ given by

$$
\nu(E)=\int_{D} \nu_{x}\left(F_{x} \cap E\right) d \mu(x) \quad \text { for Borel } E \subset \mathbb{R}^{2}
$$

extends to a Radon measure on the plane. 
We give the proof of this result after we prove the measurability of the various maps that are used in its proof.

Let $B$ be a (non-empty) compact set that is disjoint from the non-empty compact set $\Gamma$, and let $\delta=\inf \{|x-u|: x \in B, u \in \Gamma\}$, and so $\delta>0$. Let $I=[\delta$, diam $(B \cup \Gamma)]$. Letting $S^{1}$ denote the unit circle, we define $K \subseteq B \times S^{1} \times I$ by

$$
K=\left\{(x, \theta, t) \in B \times S^{1} \times \mathbb{R}^{+}: x+t \hat{\theta} \in \Gamma\right\},
$$

where $\hat{\theta}=(\cos \theta, \sin \theta)$. Notice that $K$ is compact and a lifting of $\Gamma$.

For $x \in B$ and $\theta \in S^{1}$, define $\gamma: B \times S^{1} \rightarrow I \cup\{\infty\}$ by

$$
\gamma(x, \theta)= \begin{cases}\inf \{t>0: x+t \hat{\theta} \in \Gamma\} & \text { if }\left(x+\hat{\theta} \mathbb{R}^{+}\right) \cap \Gamma \neq \emptyset \\ \infty & \text { otherwise. }\end{cases}
$$

Observe that $x+\gamma(x, \theta) \hat{\theta} \in \Gamma$ for any $x \in B$ and $\theta \in S^{1}$ for which $\left(x+\hat{\theta} \mathbb{R}^{+}\right) \cap \Gamma \neq \emptyset$.

If we define

$$
\operatorname{gr}(\gamma)=\left\{(x, \theta, \gamma(x, \theta)):(x, \theta) \in B \times S^{1}, \gamma(x, \theta)<\infty\right\},
$$

then $\operatorname{gr}(\gamma) \subseteq K$.

Lemma 3.2. The function $\gamma$ is lower semicontinuous. In particular, $\operatorname{gr}(\gamma)$ is a $G_{\delta}$-subset of $K$.

Proof. Since the graph of $\gamma$ is the lower envelope of the compact set $K$, it follows that $\gamma$ is lower semicontinuous. It is a standard result that the graph of a Baire-1 function is $G_{\delta}$; see, for example, [5, Ch. II, $\S 31$, VII, Theorem 1].

For $C \subseteq K$ and $x \in B$, define $C_{x}=C \cap\left(\{x\} \times S^{1} \times I\right)$, the slice of $C$ through $x$. For convenience, we let $\operatorname{gr}_{x}(\gamma)$ denote $(\operatorname{gr}(\gamma))_{x}$.

Recall that $\mathcal{M}(K)$ denotes the set of Radon measures supported in $K$. The set $\mathcal{M}(K)$ can be given the topology of weak convergence by using, as a base, sets of the form

$$
\left\{\mu \in \mathcal{M}(K): \int f d \mu<a\right\},
$$

where $a \in \mathbb{R}$ and $f \in C(K)$, the set of real-valued continuous functions on $K$. With this topology, $\mathcal{M}(K)$ is a Polish space; see [6, §14.15] and [4, II.17].

Lemma 3.3. Let $E$ be a Borel subset of $K$. Then the functions $F_{E}: \mathcal{M}(K) \rightarrow \mathbb{R}$ and $G_{E}: B \times \mathcal{M}(K) \rightarrow \mathbb{R}$ given by

$$
F_{E}(\nu)=\nu(E) \text { and } G_{E}(x, \nu)=\nu\left(E_{x}\right)
$$

are Borel. In particular, $\left\{(x, \nu) \in B \times \mathcal{M}(K): \nu\left(E_{x}\right)>0\right\}$ is a Borel set.

Proof. Let $E \subseteq K$ be a Borel set. We show that $G_{E}$ is Borel; the proof that $F_{E}$ is Borel is similar.

Suppose first that $E$ is a compact subset of $K$. Then for $x \in B, E_{x}$ is also compact, and for $\mu \in \mathcal{M}(K)$,

$$
G_{E}(x, \nu)=\nu\left(E_{x}\right)<c
$$

if and only if

there is $f \in C^{+}(K)$ such that $f>1$ on $E_{x}$ and $\int f d \nu<c$. 
(Here $C^{+}(K)$ denotes the set of non-negative real-valued continuous functions on $K$.) For a given $f \in C^{+}(K)$, the sets

$$
B_{f}=\left\{x \in B: f>1 \text { on } E_{x}\right\} \text { and } M_{f}=\left\{\nu \in \mathcal{M}(K): \int f d \nu<c\right\}
$$

are open subsets of $B$ and $\mathcal{M}(K)$, respectively. Hence

$$
\left\{(x, \nu): \nu\left(E_{x}\right)<c\right\}=\bigcup_{f \in C^{+}(K)} B_{f} \times M_{f}
$$

is an open set, and so $G_{E}$ is upper semi-continuous, and in particular, Borel.

If $E_{1} \subseteq E_{2} \subseteq E_{3} \subseteq \cdots$ is an increasing sequence of compact sets, and $G_{1}, G_{2}$, $G_{3}, \ldots$ the associated sequence of maps, then

$$
G_{\cup_{i \in \mathbb{N}} E_{i}}=\lim _{i \rightarrow \infty} G_{i}
$$

is a Borel map. Similarly, if $E_{1} \supseteq E_{2} \supseteq E_{3} \supseteq \cdots$ is a decreasing sequence of compact sets, and $G_{1}, G_{2}, G_{3}, \ldots$ the associated sequence of maps, then

$$
G_{\cap_{i \in \mathbb{N}} E_{i}}=\lim _{i \rightarrow \infty} G_{i}
$$

is also a Borel map. Hence for a general Borel set $E$, the map $G_{E}$ is Borel.

Lemma 3.4. Let $E$ be a Borel subset of $K$. The set

$$
\left\{(x, \nu) \in B \times \mathcal{M}(K): \nu\left(K \backslash\left(\operatorname{gr}_{x}(\gamma) \cap E\right)\right)=0\right\}
$$

is Borel in $B \times \mathcal{M}(K)$.

Proof. Observe that

$$
\begin{aligned}
& \left\{(x, \nu) \in B \times \mathcal{M}(K): \nu\left(K \backslash\left(\operatorname{gr}_{x}(\gamma) \cap E\right)\right)=0\right\} \\
& =\left\{(x, \nu): \nu\left(\left(\operatorname{gr}_{x}(\gamma) \cap E\right)\right)=\nu(K)\right\} \\
& =\left\{(x, \nu): \nu\left((\operatorname{gr}(\gamma) \cap E)_{x}\right)-\nu(K)=0\right\} \\
& =\left\{(x, \nu): G_{\operatorname{gr}(\gamma) \cap E}(x, \nu)-F_{K}(\nu)=0\right\} .
\end{aligned}
$$

But by Lemma 3.3. both $F_{K}$ and $G_{\operatorname{gr}(\gamma) \cap E}$ are Borel functions, and so the set is Borel.

Define a continuous function $\Pi: B \times S^{1} \times I \rightarrow \mathbb{R}^{2}$ by $\Pi(x, \theta, t)=x+t \hat{\theta}$.

Lemma 3.5. For $x \in B$, if $A \subseteq K \cap\left(\{x\} \times S^{1} \times I\right)$, then

$$
\operatorname{dim}_{H}(\Pi(A))=\operatorname{dim}_{H}(A) .
$$

Proof. This follows from the fact that $\Pi$ is bi-Lipschitz when restricted to $\{x\} \times$ $S^{1} \times I$.

In particular, since

$$
\Gamma_{x}=\Pi\left(\left(\{x\} \times S^{1} \times I\right) \cap \operatorname{gr}(\gamma)\right)=\Pi\left(\operatorname{gr}_{x}(\gamma)\right),
$$

it follows that $\operatorname{dim}_{H}\left(\Gamma_{x}\right)=\operatorname{dim}_{H}\left(\operatorname{gr}_{x}(\gamma)\right)$ for each $x \in B$. On recalling that for $A \subseteq K$ and $s \in \mathbb{R}$,

$$
\mathcal{M}^{s}(A)=\left\{\nu \in \mathcal{M}(K): \nu(A)>0 \text { and } \nu(B(\zeta, r)) \leq r^{s} \text { for } \zeta \in K \text { and } r \in(0,1]\right\} \text {, }
$$

it is an easy calculation, which we omit, to check that $\mathcal{M}^{s}(K)$ is a Borel set. Since $\operatorname{gr}_{x}(\gamma)$ is a Borel set, $\operatorname{dim}_{H}\left(\operatorname{gr}_{x}(\gamma)\right)=\sup \left\{\sigma: \mathcal{M}^{\sigma}\left(\operatorname{gr}_{x}(\gamma)\right) \neq \emptyset\right\}$. 
Proposition 3.6. If $U$ is a Borel subset of the plane, then for each $t \geq 0,\{x \in$ $\left.B: \operatorname{dim}_{H}\left(\Gamma_{x} \cap U\right)>t\right\}$ is an analytic set.

Proof. Let $E \subseteq K$ be given by $E=\Pi^{-1}(U) \cap K$, and observe that $\operatorname{gr}(\gamma) \cap E$ is a Borel subset of $K$. For $t \geq 0$,

$$
\begin{aligned}
\left\{x: \operatorname{dim}_{H}\left(\Gamma_{x} \cap U\right)>t\right\} & =\left\{x: \operatorname{dim}_{H}\left((\operatorname{gr}(\gamma) \cap E)_{x}\right)>t\right\} \\
& =\left\{x: \mathcal{M}^{\tau}\left((\operatorname{gr}(\gamma) \cap E)_{x}\right) \neq \emptyset \text { for some } \tau>t\right\} \\
& =\bigcup_{p \in \mathbb{Q}^{+}}\left\{x: \mathcal{M}^{t+p}\left((\operatorname{gr}(\gamma) \cap E)_{x}\right) \neq \emptyset\right\} .
\end{aligned}
$$

However, if $\pi_{B}: B \times \mathcal{M}(K) \rightarrow B$ denotes coordinate projection onto $B$, then

$$
\begin{gathered}
\left\{x: \mathcal{M}^{t+p}\left((\operatorname{gr}(\gamma) \cap E)_{x}\right) \neq \emptyset\right\}=\pi_{B}\left(\left\{(x, \nu): x \in B, \nu \in \mathcal{M}^{t+p}\left((\operatorname{gr}(\gamma) \cap E)_{x}\right)\right\}\right) \\
=\pi_{B}\left(\left\{(x, \nu) \in B \times \mathcal{M}^{t+p}(K): \nu\left((\operatorname{gr}(\gamma) \cap E)_{x}\right)>0\right\}\right) .
\end{gathered}
$$

Hence Lemmas 3.2 and 3.3 together imply that $\left\{x: \mathcal{M}^{t+p}\left((\operatorname{gr}(\gamma) \cap E)_{x}\right) \neq \emptyset\right\}$ is the coordinate-wise projection of a Borel set from a product of Polish spaces, and so it is analytic; see [4, Chapter III]. Hence $\left\{x: \operatorname{dim}_{H}\left(\Gamma_{x} \cap U\right)>t\right\}$ is also analytic.

Our next result is a selection theorem and allows us to choose, in a measurable way, an element of $\mathcal{M}^{t}\left(\operatorname{gr}_{x}(\gamma)\right)$ whenever $x \in B$ is such that $\operatorname{dim}_{H}\left(\Gamma_{x}\right)>t$.

Proposition 3.7. Let $t \geq 0$ and $U$ be a Borel subset of the plane. There is a map

$$
\begin{aligned}
\omega:\left\{x \in B: \operatorname{dim}_{H}\left(\Gamma_{x} \cap U\right)>t\right\} & \rightarrow \mathcal{M}(K) \\
x & \mapsto \omega_{x}
\end{aligned}
$$

such that:

(1) $\omega$ is $\sigma(\mathbf{A})$-measurable,

(2) $\omega_{x} \in \mathcal{M}^{t}(K)$, and

(3) $\omega_{x}\left(\operatorname{gr}_{x}(\gamma) \cap \Pi^{-1}(U)\right)>0$ and $\omega_{x}\left(K \backslash\left(\operatorname{gr}{ }_{x}(\gamma) \cap \Pi^{-1}(U)\right)\right)=0$ for each $x$.

(Here $\sigma(\mathbf{A})$ denotes the $\sigma$-algebra generated by the analytic sets in $B$.)

In particular, $\omega$ is $\mu$-measurable for every Radon measure $\mu$ on $B$.

Proof. Let $E=\Pi^{-1}(U) \cap K$, a Borel set. Since

$\left(B \times \mathcal{M}^{t}(K)\right) \cap\left\{(x, \nu): \nu\left((\operatorname{gr}(\gamma) \cap E)_{x}\right)>0\right\} \cap\left\{(x, \nu): \nu\left(K \backslash(\operatorname{gr}(\gamma) \cap E)_{x}\right)=0\right\}$

is Borel in $B \times \mathcal{M}(K)$, claims (1), (2), and (3) follow from the Jankov-von Neumann Uniformisation Theorem; see [4, Theorem 18.1].

Lusin's Theorem that analytic sets are universally measurable [4, Theorem 21.10] implies that sets in $\sigma(\mathbf{A})$ are also universally measurable.

We can now prove Proposition 3.1.

Proof of Proposition 3.1. Let $E=\left\{x \in C: \operatorname{dim}_{H}\left(\Gamma_{x} \cap U\right)>s\right\}$. From Proposition 3.6 (with $B=C$ ), we see that $E$ is an analytic set. Hence by Theorem 2.1 . since $\operatorname{dim}_{H}(E)>t$, there is a Radon measure $\mu$ and a compact set $B \subseteq C$ such that $0<\mu(B) \leq 1$, and for each $x \in \mathbb{R}^{2}$ and $r>0$,

$$
\mu(B(x, r)) \leq r^{t} .
$$

(Hence for any non-empty compact subset $D$ of $B$, claim 1 of Proposition 3.1 holds.) 
Proposition 3.7 enables us to find a $\sigma(\mathbf{A})$-measurable function

$$
\begin{aligned}
\omega: B & \rightarrow \mathcal{M}^{s}(K) \\
x & \mapsto \omega_{x}
\end{aligned}
$$

(where $K=\left\{(x, \theta, t) \in B \times S^{1} \times I: x+t \hat{\theta} \in \Gamma\right\}=\Pi^{-1}(\Gamma)$ ) such that

$\omega_{x}\left(\operatorname{gr}_{x}(\gamma) \cap \Pi^{-1}(U)\right)>0$ and $\omega_{x}\left(K \backslash\left(\operatorname{gr}_{x}(\gamma) \cap \Pi^{-1}(U)\right)\right)=0$, for each $x \in B$.

Moreover, there is a constant $c$ such that $\omega_{x}(K) \leq c$ for all $x \in B$.

For Borel $E \subseteq K$, define

$$
\Omega(E)=\int_{B} \omega_{x}(E) d \mu(x) .
$$

We omit the routine verification that $\Omega$ extends to a Radon measure on $K$ on setting $\Omega^{*}(A)=\inf \{\Omega(E): A \subseteq E$ and $E$ is Borel $\}$ for non-Borel sets $A$. Now

$$
\Omega\left(\operatorname{gr}(\gamma) \cap \Pi^{-1}(U)\right)>0
$$

and

$$
\Omega\left(K \backslash\left(\operatorname{gr}(\gamma) \cap \Pi^{-1}(U)\right)\right)=0 .
$$

Hence there is a compact set $K^{(1)} \subseteq \operatorname{gr}(\gamma) \cap \Pi^{-1}(U)$ for which $\Omega\left(K^{(1)}\right)>0$ and for each $x \in \pi\left(K^{(1)}\right), \omega_{x}\left(K^{(1)}\right)>0$. (Here $\pi$ denotes projection onto the first coordinate, $(x, r, \theta) \mapsto x$.) For $x \in \pi\left(K^{(1)}\right)$, let

$$
E_{x}=\Pi\left(\left(K^{(1)}\right)_{x}\right)=\Pi\left(K^{(1)} \cap \Pi^{-1}(U) \cap \operatorname{gr}_{x}(\gamma)\right) .
$$

Notice that there is a constant $d \geq 1$ so that for each $x \in B$, any $u \in \Gamma$ and any $r>0$

$$
\Pi^{-1}(B(u, r))_{x} \subseteq B\left(\Pi^{-1}(u)_{x}, d r\right) .
$$

Hence if, for $x \in B$, we define a Radon measure $\nu_{x}$ on $\Gamma$ by

$$
\nu_{x}(A)=d^{-s} \omega_{x}\left(\Pi^{-1}(A)\right)=d^{-s} \omega_{x}\left(\left(\Pi^{-1}(A \cap U)\right)_{x}\right), \text { for } A \subseteq \mathbb{R}^{2},
$$

then for each $x \in B, 0<\nu_{x}\left(\mathbb{R}^{2}\right) \leq c d^{-s} \leq c, \nu_{x}\left(\mathbb{R}^{2} \backslash\left(\Gamma_{x} \cap U\right)\right)=0$ and for each $u \in \Gamma$ and $0<r \leq 1, \nu_{x}(B(u, r)) \leq r^{s}$. Hence for each $x \in B$, claim (2)(a) holds for $\nu_{x}$. Moreover, $x \mapsto \nu_{x}$ is a $\sigma(\mathbf{A})$-measurable function.

It remains to show that claims (2)(b) and (c) hold for the measures $\nu_{x}$ and sets $E_{x}$ provided that we make a suitable choice of sets $D$ and $F_{x}$. Since $0<\xi<s-1$, by applying Lemma 2.6 to the measures $\left.\nu_{x}\right|_{E_{x}}$, we find that for each $x \in \pi\left(K^{(1)}\right)$ and for $\left.\nu_{x}\right|_{E_{x}}$-a.e. $u \in \Gamma_{x} \cap U$,

$$
\min \left\{\liminf _{r \searrow 0} \frac{\nu_{x}\left(E_{x} \cap T_{x}^{+}(u, r)\right)}{r^{1+\xi}}, \liminf _{r \searrow 0} \frac{\nu_{x}\left(E_{x} \cap T_{x}^{-}(u, r)\right)}{r^{1+\xi}}\right\}=+\infty .
$$

That is, for each $x \in \pi\left(K^{(1)}\right)$ and $\omega_{x}$-a.e. $\zeta \in K^{(1)}$,

$$
\begin{aligned}
& \liminf _{r \searrow 0} \frac{\omega_{x}\left(K^{(1)} \cap \Pi^{-1}\left(T_{x}^{+}(\Pi(\zeta), r)\right)\right)}{r^{1+\xi}} \\
&=\liminf _{r \searrow 0} \frac{\omega_{x}\left(K^{(1)} \cap \Pi^{-1}\left(T_{x}^{-}(\Pi(\zeta), r)\right)\right)}{r^{1+\xi}}=+\infty .
\end{aligned}
$$

It is routine to verify that $f: K^{(1)} \rightarrow \mathbb{R} \cup\{+\infty\}$ given by

$$
f(x, \theta, t)=\min \left\{\liminf _{r \searrow 0} \frac{\nu_{x}\left(E_{x} \cap T_{x}^{+}(x+t \hat{\theta}, r)\right)}{r^{1+\xi}}, \liminf _{r \searrow 0} \frac{\nu_{x}\left(E_{x} \cap T_{x}^{-}(x+t \hat{\theta}, r)\right)}{r^{1+\xi}}\right\}
$$


is a universally measurable function, and so $K_{\infty}^{(1)}=\left\{\zeta \in K^{(1)}: f(\zeta)=+\infty\right\}$ is a universally measurable set with $\omega_{x}\left(K^{(1)} \backslash K_{\infty}^{(1)}\right)=0$ for all $x \in \pi\left(K^{(1)}\right)$. Hence $m^{*}\left(K^{(1)} \backslash K_{\infty}^{(1)}\right)=0$. Now

$$
K_{\infty}^{(1)}=\bigcap_{M \in \mathbb{N}} \bigcup_{n \in \mathbb{N}} K_{M, n}^{(1)}
$$

where

$$
\begin{aligned}
K_{M, n}^{(1)}=\{(x, \theta, t) & \in K^{(1)}: \text { if } r \in\left(0, \frac{1}{n}\right], \text { then } \\
& \left.\min \left\{\nu_{x}\left(E_{x} \cap T^{+}(x+t \hat{\theta}, r)\right), \nu_{x}\left(E_{x} \cap T^{-}(x+t \hat{\theta}, r)\right)\right\}>M r^{1+\xi}\right\} .
\end{aligned}
$$

Thus we can find $M, n \in \mathbb{N}$ for which $m^{*}\left(K_{M, n}^{(1)}\right)>0$, and so we can find a compact set $K^{(2)} \subseteq K_{M, n}^{(1)}$ with $m^{*}\left(K^{(2)}\right)>0$. It follows that there is $p>0$ and a compact set $D \subseteq \pi\left(K^{(2)}\right) \subseteq B$ for which $\mu(D)>0$ and for all $x \in D$, we have $\omega_{x}\left(K^{(2)}\right)>p$. For $x \in D$, let

$$
F_{x}=\Pi\left(\left(K^{(2)}\right)_{x}\right) \subseteq \Gamma_{x} \cap U
$$

and notice that $F_{x}$ is a compact subset of $E_{x}$ with $\nu_{x}\left(F_{x}\right)=\omega_{x}\left(K^{(2)}\right)>p$, and for $u \in F_{x}$,

On defining

$$
\min \left\{\nu_{x}\left(E_{x} \cap T^{+}(u, r)\right), \nu_{x}\left(E_{x} \cap T^{-}(u, r)\right)\right\}>M r^{1+\xi} .
$$

$$
\nu(E)=\int_{D} \nu_{x}\left(F_{x} \cap E\right) d \mu(x) \text { for Borel sets } E,
$$

and extending to non-Borel sets in the usual way, the proposition follows.

\section{Proof of Theorem 1.2}

We now draw all our preparatory work together and prove Theorem 1.2 ,

Let $\Gamma$ be a compact connected subset of the plane for which $1<\operatorname{dim}_{H}(\Gamma) \leq 2$. If $\operatorname{dim}_{H}(\Gamma)=2$, then set $d=2$; otherwise choose $\operatorname{dim}_{H}(\Gamma)<d<2$. Notice that, in either case, if $\nu$ is a non-zero Radon measure supported in $\Gamma$, then

$$
I_{d}(\nu)=\iint|x-y|^{-d} d \nu(x) d \nu(y)=+\infty
$$

(If $d=2$, then, since $\mathcal{H}^{2}(\Gamma)<\infty$, Theorem 8.7 of [6] implies that $I_{2}(\nu)=+\infty$.)

It is enough for us to show that whenever $C$ is a closed ball disjoint from $\Gamma$ with diameter sufficiently small compared with its distance to $\Gamma$, and $U$ is a sufficiently small open set, then

$$
\operatorname{dim}_{H}\left(\left\{x \in C: \operatorname{dim}_{H}\left(\Gamma_{x} \cap U\right)>s\right\}\right) \leq \frac{d-s}{s-1} .
$$

So let $C$ be a closed ball disjoint from $\Gamma$, and let $U$ be an open set meeting $\Gamma$, and suppose that they both have small enough diameter such that

$$
2 \operatorname{diam}(C)<\inf \{|x-u|: x \in C, u \in \Gamma\}=d_{-} \text {, say, }
$$

and

$$
\left\langle(u-x)^{\wedge},(u-y)^{\wedge}\right\rangle \in\left[\frac{1}{2}, 1\right] \text { for each } u \in U \text { and each } x, y \in C .
$$

If $x, y \in C$, then $2|x-y| \leq d_{-} \leq \min \{d(x, \Gamma), d(y, \Gamma)\}$. Let $d_{+}=\operatorname{diam}(C)+$ $\operatorname{diam}(\Gamma)+\operatorname{dist}(C, \Gamma)$, and so

$$
2|x-y| \leq d_{-} \leq \min \{d(x, \Gamma), d(y, \Gamma)\} \leq \max \{d(x, \Gamma), d(y, \Gamma)\}+\operatorname{diam}(\Gamma) \leq d_{+} .
$$


Now suppose that $t>0$ is such that

$$
\operatorname{dim}_{H}\left(\left\{x \in C: \operatorname{dim}_{H}\left(\Gamma_{x} \cap U\right)>s\right\}\right)>t ;
$$

we aim to show that $t \leq(d-s) /(s-1)$.

Let $\xi \in(0, s-1)$. By Proposition 3.1] we can find $M, p, q>0$, a compact set $D \subseteq C$ and a Radon measure $\mu$ with $0<\mu(D) \leq 1$ such that:

(1) for each $x \in D$ and $r>0, \mu(B(x, r)) \leq r^{t}$;

(2) for each $x \in D$, there is a Radon measure $\nu_{x}$ and compact sets $F_{x} \subseteq E_{x} \subseteq$ $U \cap \Gamma_{x}$ such that:

(a) $\nu_{x}\left(\mathbb{R}^{2} \backslash \Gamma_{x}\right)=0, \nu_{x}\left(\mathbb{R}^{2}\right) \leq c$ and for $0<r \leq 1$ and $u \in \Gamma, \nu_{x}(B(u, r)) \leq$ $r^{s}$

(b) $\nu_{x}\left(F_{x}\right)>p$;

(c) for $0<r \leq q$ and $u \in F_{x}$,

$$
\min \left\{\nu_{x}\left(T_{x}^{+}(u, r) \cap E_{x}\right), \nu_{x}\left(T_{x}^{-}(u, r) \cap E_{x}\right)\right\}>M r^{1+\xi} .
$$

Moreover $\nu$ given by

$$
\nu(E)=\int_{D} \nu_{x}\left(F_{x} \cap E\right) d \mu(x) \text { for Borel } E \subset \mathbb{R}^{2}
$$

extends to a non-zero Radon measure on the plane.

Let $A, B \subseteq \mathbb{R}^{2}$ be compact and suppose that $\psi \in(0,1 / 2)$ is such that for $u \in\left(A \cap F_{x}\right) \cup\left(B \cap F_{y}\right)$,

$$
\left|\left\langle(u-x)^{\wedge},(u-y)^{\wedge}\right\rangle\right| \in[1 / 2,1-\psi] .
$$

Then all the hypotheses of Propositions 2.8 and 2.9 are satisfied (after suitable relabelling - in particular, replacing $F_{x}$ by $A \cap F_{x}$ and $F_{y}$ by $B \cap F_{y}$ ) and so, for

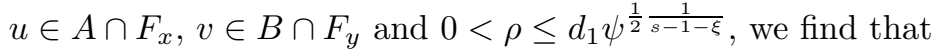

$$
\nu_{y}\left(A \cap F_{y} \cap B(u, \rho)\right) \leq c_{1} \psi^{-\frac{1}{2} \frac{s-1}{2+\xi-s}} \rho^{\frac{1+\xi}{2+\xi-s}}
$$

and for $0<\rho \leq d_{2} \psi^{\frac{1}{2} \frac{1}{s-1-\xi}}$,

$$
\begin{aligned}
\left(\nu_{x} \otimes \nu_{y}\right)\left(\left(F_{x} \times F_{y}\right)\right. & \cap(A \times B) \cap\{(u, v):|u-v| \leq \rho\}) \\
\leq & c_{2} \operatorname{arc-diam}_{\frac{1}{2}(x+y)}\left(A \cap F_{x} \cap B\left(F_{y} \cap B, \rho\right)\right)\left(\psi^{-\frac{1}{2}} \rho\right)^{\frac{s+\xi}{2+\xi-s}} .
\end{aligned}
$$

We now pull all our estimates together and explicitly calculate the $d$-energy of the measure $\nu$ given in equation (4.1). Noting that for $\tau>0$,

$$
\int|u-v|^{-\tau} d(\nu \times \nu)(u, v)=\int_{D \times D} \int_{F_{x} \times F_{y}}|u-v|^{-\tau} d\left(\nu_{x} \times \nu_{y}\right)(u, v) d(\mu \times \mu)(x, y),
$$

and, since $I_{d}(\nu)=\int|u-v|^{-d} d(\nu \times \nu)(u, v)=+\infty$, we know that

$$
\int_{D \times D} \int_{F_{x} \times F_{y}}|u-v|^{-d} d\left(\nu_{x} \otimes \nu_{y}\right)(u, v) d(\mu \otimes \mu)(x, y)=+\infty .
$$

Now fix $x \neq y \in D$. We aim to estimate the inner integral of (4.4) using equations (4.2) and (4.3). In order to reduce writing, we translate so that $\frac{1}{2}(x+y)=$ 0 and let $a=y$, so $|x-y|=2|a|$. 


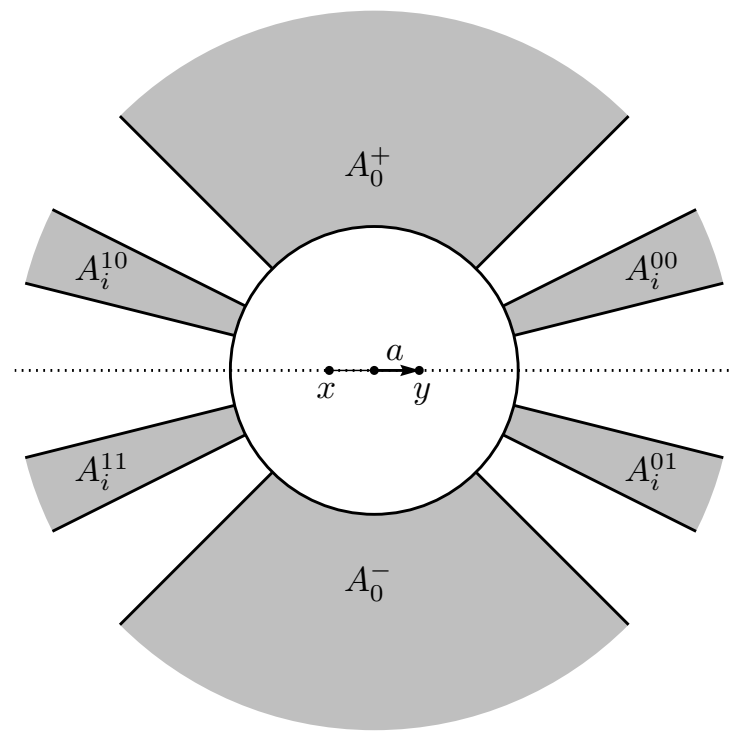

Figure 6. $A_{i}^{m n}$

Using Fubini's theorem, we find that

$$
\begin{aligned}
& \int_{F_{x} \times F_{y}}|u-v|^{-d} d\left(\nu_{x} \otimes \nu_{y}\right)(u, v) \\
& =\int_{0}^{\infty}\left(\nu_{x} \otimes \nu_{y}\right)\left(\left\{(u, v) \in F_{x} \times F_{y}:|u-v|^{-d} \geq r\right\}\right) d r \\
& =d \int_{0}^{\infty} \rho^{-d-1}\left(\nu_{x} \otimes \nu_{y}\right)\left(\left\{(u, v) \in F_{x} \times F_{y}:|u-v| \leq \rho\right\}\right) d \rho \\
& =d \int_{F_{x}} \int_{0}^{\infty} \rho^{-d-1} \nu_{y}\left(F_{y} \cap B(u, \rho)\right) d \rho d \nu_{x}(u) .
\end{aligned}
$$

We now split up the plane into regions where we can estimate the 'angle' $\psi$ subtended by points in the region with 0 and $a$. Let

$$
\begin{gathered}
A_{0}^{+}=\left\{w \in A\left(0, d_{-}, d_{+}\right):\left\langle w, a^{\perp}\right\rangle \geq|\langle w, a\rangle|\right\}, \\
A_{0}^{-}=\left\{w \in A\left(0, d_{-}, d_{+}\right):\left\langle w, a^{\perp}\right\rangle \leq-|\langle w, a\rangle|\right\},
\end{gathered}
$$

and for $m, n \in\{0,1\}$ and $i \in \mathbb{N}$, set

$$
\begin{array}{r}
A_{i}^{m n}=\left\{w \in A\left(0, d_{-}, d_{+}\right):\left|\left\langle w, a^{\perp}\right\rangle /\langle w, a\rangle\right| \in\left[2^{-i}, 2^{1-i}\right],\right. \\
\left.(-1)^{n}\left\langle w, a^{\perp}\right\rangle>0 \text { and }(-1)^{m}\langle w, a\rangle>0\right\},
\end{array}
$$

noting that

$$
\operatorname{arc}_{\operatorname{diam}}\left(A_{i}^{m n}\right) \preceq 2^{-i} .
$$

(Here, and subsequently, we let $\preceq$ denote inequality up to a finite positive constant that is independent of $x$ and $y$.) See Figure 6.

Observe that Lemma 2.3 implies that for $w \in A_{i}^{m n}$,

$$
\frac{3}{5} \leq \frac{\langle w-a, w+a\rangle}{|w-a||w+a|} \leq 1-\frac{9}{17 d_{+}^{2}}\left(|a| 2^{-i}\right)^{2}
$$


and for $w \in A_{0}^{+} \cup A_{0}^{-}$,

$$
\frac{3}{5} \leq \frac{\langle w-a, w+a\rangle}{|w-a||w+a|} \leq 1-\frac{9}{17 d_{+}^{2}}|a|^{2} .
$$

For $i \in\{0\} \cup \mathbb{N}$, let $\psi_{i}=\left(\frac{2}{5} \frac{d_{-}}{d_{2}}|a| 2^{-i}\right)^{2}$ and let $\rho_{i}=d_{2} \psi_{i}^{\frac{1}{2} \frac{1}{s-1-\xi}}$. Observe that if $u \in A_{i}^{m n}$ and $|u-v| \leq \rho_{i}$ with $v \in A\left(0, d_{-}, d_{+}\right)$, then $v \in A_{i-1}^{m n} \cup A_{i}^{m n} \cup A_{i+1}^{m n}$, since $\rho_{i} \leq \frac{2}{5} d_{-} 2^{-i}$. Similarly, if $u \in A_{0}^{+} \cup A_{0}^{-}$, and $v \in A\left(0, d_{-}, d_{+}\right)$with $|u-v| \leq \rho_{0}$, then $v \in A_{1}^{m n}$ for some choice of $m$ and $n$.

Writing $f(\rho)=\rho^{-d-1} \nu_{y}\left(F_{y} \cap B(u, \rho)\right)$, we let

$$
I_{0}^{ \pm}=\int_{F_{x} \cap A_{0}^{ \pm}} \int_{0}^{\infty} f(\rho) d \rho d \nu_{x}(u) \quad \text { and } \quad I_{i}^{m n}=\int_{F_{x} \cap A_{i}^{m n}} \int_{0}^{\infty} f(\rho) d \rho d \nu_{x}(u) .
$$

We must estimate (4.5), and so must estimate

$$
\begin{aligned}
& \int_{F_{x}} \int_{0}^{\infty} \rho^{-d-1} \nu_{y}\left(F_{y} \cap B(u, \rho)\right) d \rho d \nu_{x}(u) \\
& =\left(\int_{F_{x} \cap A_{0}^{+}}+\int_{F_{x} \cap A_{0}^{-}}+\sum_{m, n=0}^{1} \sum_{i=1}^{\infty} \int_{F_{x} \cap A_{i}^{m n}}\right) \int_{0}^{\infty} f(\rho) d \rho d \nu_{x}(u) \\
& =I_{0}^{+}+I_{0}^{-}+\sum_{m, n=0}^{1} \sum_{i=1}^{\infty} I_{i}^{m n}
\end{aligned}
$$

However,

$$
\begin{aligned}
I_{i}^{m n} & =\int_{F_{x} \cap A_{i}^{m n}}\left(\int_{0}^{\rho_{i}}+\int_{\rho_{i}}^{\infty}\right) f(\rho) d \rho d \nu_{x}(u) \\
& =\int_{F_{x} \cap A_{i}^{m n} \cap B\left(F_{y} \cap B\left(A_{i}^{m n}, \rho_{i}\right), \rho_{i}\right)} \int_{0}^{\rho_{i}} f(\rho) d \rho d \nu_{x}(u)+\int_{F_{x} \cap A_{i}^{m n}} \int_{\rho_{i}}^{\infty} f(\rho) d \rho d \nu_{x}(u) \\
& =I_{i, 1}^{m n}+I_{i, 2}^{m n}, \text { say. }
\end{aligned}
$$

Lemma 4.1. Suppose that $V \subseteq A\left(0, d_{-}, d_{+}\right)$and $0<r<1$. Then

$$
\int_{F_{x} \cap V} \int_{r}^{\infty} f(\rho) d \rho d \nu_{x}(u) \preceq r^{s-d} \operatorname{arc-diam}\left(F_{x} \cap V\right)^{s-1} .
$$

Proof. Using the crude estimate that for $u \in F_{x} \cap V, \nu_{y}\left(F_{y} \cap B(u, \rho)\right) \leq \min \left\{c, 2^{s} \rho^{s}\right\}$ together with Lemma 2.4, we find that

$$
\begin{aligned}
\int_{F_{x} \cap V} \int_{r}^{\infty} f(\rho) d \rho d \nu_{x}(u) & \leq\left(\frac{2^{s}}{d-s} r^{s-d}+\frac{2^{d}}{d} c^{1-d / s}\right) \nu_{x}\left(F_{x} \cap V\right) \\
& \preceq r^{s-d} \operatorname{arc-diam}\left(F_{x} \cap V\right)^{s-1} .
\end{aligned}
$$

In particular, Lemma 4.1 implies that

$$
I_{i, 2}^{m n} \preceq \rho_{i}^{s-d} \operatorname{arc-diam}_{0}\left(F_{x} \cap A_{i}^{m n}\right)^{s-1} \preceq|a|^{\frac{s-d}{s-1-\xi}} 2^{-\left(\frac{s-d}{s-1-\xi}+s-1\right) i} .
$$

In order to estimate $I_{i, 1}^{m n}$, equation (4.3), Fubini's theorem and the fact that if $u \in F_{x} \cap A_{i}^{m n}$ and $v \in B\left(u, \rho_{i}\right) \cap F_{y}$, then $v \in A_{i-1}^{m n} \cup A_{i}^{m n} \cup A_{i+1}^{m n}$ are used to 
calculate that, provided $\frac{s+\xi}{2+\xi-s}-d>0$,

$$
\begin{aligned}
& I_{i, 1}^{m n} \\
& =\int_{0}^{\rho_{i}} \rho^{-d-1}\left(\nu_{x} \otimes \nu_{y}\right)\left\{(u, v) \in\left(F_{x} \cap A_{i}^{m n}\right) \times\left(F_{y} \cap B\left(A_{i}^{m n}, \rho\right)\right):|u-v| \leq \rho\right\} d \rho \\
& \leq c_{2} \operatorname{arc-diam}_{0}\left(A_{i}^{m n} \cap F_{x}\right) \int_{0}^{\rho_{i}} \rho^{-d-1}\left(\psi_{i+1}^{-\frac{1}{2}} \rho\right)^{\frac{s+\xi}{2+\xi-s}} d \rho \\
& \preceq \operatorname{arc-diam}_{0}\left(A_{i}^{m n} \cap F_{x}\right) \psi_{i+1}^{-\frac{1}{2} \frac{s+\xi}{2+\xi-s}} \rho_{i}^{\frac{s+\xi}{2+\xi-s}-d} \\
& \preceq \operatorname{arc-diam}_{0}\left(A_{i}^{m n} \cap F_{x}\right) \psi_{i}^{\frac{1}{2}\left(\frac{s+\xi-d}{s-1-\xi}\right)} \\
& \preceq|a|^{\frac{s+\xi-d}{s-1-\xi}} 2^{-i\left(\frac{2 s-1-d}{s-1-\xi}\right)} .
\end{aligned}
$$

Combining these estimates for $I_{i, 1}^{m n}$ and $I_{i, 2}^{m n}$, we deduce that, provided $\frac{s+\xi}{2+\xi-s}-$ $d>0$,

$$
\begin{aligned}
I_{i}^{m n} & \preceq|a|^{\frac{s+\xi-d}{s-1-\xi}} 2^{-i\left(\frac{2 s-1-d}{s-1-\xi}\right)}+|a|^{\frac{s-d}{s-1-\xi}}\left(2^{-i}\right)^{\frac{s-d}{s-1-\xi}+s-1} \\
& =|a|^{\frac{s-d}{s-1-\xi}}\left(\left(2^{-i}\right)^{\frac{2 s-1-d}{s-1-\xi}}+\left(2^{-i}\right)^{\frac{s-d}{s-1-\xi}+s-1}\right) .
\end{aligned}
$$

Hence

$$
\sum_{m, n=0}^{1} \sum_{i=1}^{\infty} I_{i}^{m n} \preceq|a|^{\frac{s-d}{s-1-\xi}},
$$

provided that $m=\min \left\{\frac{s+\xi}{2+\xi-s}-d, \frac{2 s-1-d}{s-1-\xi}, \frac{s-d}{s-1-\xi}+s-1\right\}>0$. Estimating $I_{0}^{+}$ and $I_{0}^{-}$in a similar way, we find that

$$
I_{0}^{+}+I_{0}^{-}+\sum_{m, n=0}^{1} \sum_{i=1}^{\infty} I_{i}^{m n} \preceq|a|^{\frac{s-d}{s-1-\xi}},
$$

provided that $m>0$. Hence,

$$
\int_{F_{x} \times F_{y}}|u-v|^{-d} d\left(\nu_{x} \otimes \nu_{y}\right)(u, v) \preceq|x-y|^{-\frac{d-s}{s-1-\xi}}, \text { provided that } m>0 .
$$

Thus, if $m>0$, then

$$
+\infty=I_{d}(\nu) \preceq I_{\frac{d-s}{s-1-\xi}}(\mu),
$$

and this gives a contradiction if $\frac{d-s}{s-1-\xi}<t$, the dimension of $\mu$. Since $0<\xi<s-1$ can be arbitrarily small, it follows that if $s>\max \left\{\frac{1}{2}(d+1), \frac{2 d}{d+1}, \frac{1}{2}+\sqrt{d-\frac{3}{4}}\right\}=$ $\frac{1}{2}+\sqrt{d-\frac{3}{4}}$, then $t \leq \frac{d-s}{s-1}$ and Theorem 1.2 (and hence Theorem 1.1) follows.

\section{REFERENCES}

[1] K. J. Falconer, The geometry of fractal sets, Cambridge University Press (1990). MR.867284 (88d:28001)

[2] H. Federer, Geometric Measure Theory, Springer-Verlag (1996).

[3] E. Järvenpää, M. Järvenpää, P. MacManus, T. C. O'Neil, Visible parts and dimensions, Nonlinearity 16 (2003), 803-818. MR1975783 (2004d:28019)

[4] A. S. Kechris, Classical Descriptive Set Theory, Graduate Texts in Math., vol. 156, SpringerVerlag (1995). MR1321597(96e:03057)

[5] K. Kuratowski, Topology, Vol. I, Academic Press (1966). MR0217751 (36:840) 
[6] P. Mattila, Geometry of sets and measures in Euclidean spaces, Cambridge University Press (1995). MR 1333890 (96h:28006)

[7] O. Nikodym, Sur les points linéairement accessibles des ensembles plans, Fundamenta Mathematicae 7 (1925), 250-258.

[8] O. Nikodym, Sur la mesure des ensembles plans dont tous les points sont rectilinéairement accessibles, Fundamenta Mathematicae 10 (1927), 116-168.

[9] O. Nikodym, Sur un ensemble plan et fermé dont les points qui sont rectilinéairement accessibles forment un ensemble non mesurable (B), Fundamenta Mathematicae 11 (1928), 239-263.

[10] C. A. Rogers, Hausdorff Measures, Cambridge University Press (1998). MR0281862 (43:7576)

[11] P. Urysohn, Problème 29, Fundamenta Mathematicae 5 (1923), 337.

[12] P. Urysohn, Sur les points accessibles des ensembles fermés, Proceedings Amsterdam 28 (1925), 984-993.

Faculty of Mathematics and Computing, The Open University, Walton Hall, Milton Keynes, MK7 6AA, United Kingdom

E-mail address: t.c.oneil@open.ac.uk 